\title{
Coloron excitations of the SU(3) Haldane-Shastry model
}

\author{
Dirk Schuricht and Martin Greiter \\ Institut für Theorie der Kondensierten Materie, \\ Universität Karlsruhe, Postfach 6980, 76128 Karlsruhe, Germany
}

\begin{abstract}
In a recent publication, we proposed two possible wave functions for the elementary excitations of the SU(3) Haldane-Shastry model (HSM), but argued on very general grounds that only one or the other can be a valid excitation. Here we provide the explicit details of our calculation proving that the wave function describing a coloron excitation which transforms according to representation $\overline{3}$ under SU(3) rotations if the spins of the original model transform according to representation 3 , is exact. We further provide an explicit construction of the exact color-polarized two-coloron eigenstates, and thereby show that colorons are free but that their relative momentum spacings are shifted according to fractional statistics with parameter $g=2 / 3$. We evaluate the $\mathrm{SU}(3)$ spin currents. Finally, we interpret our results within the framework of the asymptotic Bethe Ansatz and generalize some of them to the case of $\mathrm{SU}(n)$.
\end{abstract}

PACS numbers: 75.10.Pq, 02.30.Ik, 75.10.Jm, 71.10.Pm 


\section{INTRODUCTION}

In recent years, there has been substantial interest in models in condensed matter physics with symmetry groups larger than $\mathrm{SU}(2)$, the group underlying the usual spin algebra. In particular, transition-metal oxides [1, 2, 3, 4], where the electron spin is coupled to orbital degrees of freedom, have been described theoretically by models with SU(4) symmetry [ㅌ, 6, 7, 8, 9]. Furthermore, there has been a growing interest in the $\operatorname{SU}(n)$ generalization of the Hubbard model 10, 11] motivated by possible experimental realizations in systems of ultracold atoms [12, 13].

In a previous paper [14], we have used the $\mathrm{SU}(n)$ Haldane-Shastry model to make the case that the elementary excitations of $\mathrm{SU}(n)$ spin chains transform under the representation conjugate to the representation of the $\mathrm{SU}(n)$ spins on the chain, and only exist in one $\mathrm{n}^{\text {th }}$ of the Brillouin zone. In this article, we will present for one thing the detailed calculations underlying our line of argumentation in 14|. To this end, we focus on the SU(3) HaldaneShastry model (HSM) 15, 16, 17, 18, 19, 20, 21, 22], which serves as a paradigm for not only the $\mathrm{SU}(3)$ spin chain, but, as we shall see, also illustrates some very general properties of $\mathrm{SU}(n)$ chains. In particular, we derive the quantum numbers of the elementary, fractionally quantized excitations, the analogs of the spinon excitations for $\mathrm{SU}(2)$, which we call colorons for $\mathrm{SU}(3)$. As already mentioned, the key result is that these excitations transform under the $\mathrm{SU}(n)$ representation conjugate to the representation of the original $\mathrm{SU}(n)$ spins localized at the sites of the chain. In the case of $\mathrm{SU}(3)$, if a basis for the original spins is spanned by the colors blue, red, and green, a basis for the coloron excitations is given by the complementary colors yellow, cyan, and magenta (see Fig. 11). This result is meaningless for $\mathrm{SU}(2)$, as the representations of $\mathrm{SU}(2)$ are self-conjugate, but significant in all other instances of fractional quantization in $\mathrm{SU}(n)$ chains and possible higher dimensional liquids, regardless of model specifics. In our analysis, however, we will primarily focus on the $\mathrm{SU}(3) \mathrm{HSM}$, as this is the simplest model in which this general result can be illustrated through exact calculations, and only afterwards generalize our results to the case of $\mathrm{SU}(n)$. The other significant advancement we report here is the construction of the exact, colorpolarized two-coloron eigenstates. We obtain those through derivation and solution of a Sutherland-type equation which is similar to the case of two spinons in the SU(2) HSM. Our explicit calculation implies that the colorons are, just like the spinons, non-interacting 
or free, but that the spacings in the difference of the individual coloron momenta $p_{n}$ and $p_{m}$ with $p_{n} \geq p_{m}$ are given by $p_{n}-p_{m}=\frac{2 \pi}{N}\left(\frac{2}{3}+\right.$ integer $)$. We will argue that this spacing is a direct manifestation of the fractional statistics [23, 24] of the colorons with statistical exclusion parameter $g=2 / 3$. This value is consistent with what we find by naive state counting. We then proceed by calculating the $\mathrm{SU}(3)$ spin currents, i.e., the eigenvalues of the diagonal components of the rapidity operator $\Lambda^{3}$ and $\Lambda^{8}$, for the one- and two-coloron states. Thereafter, we interpret our results within the framework of the asymptotic Bethe Ansatz. Finally, we generalize some of our conclusions to the case of SU $(n)$. Many details of our calculations have been exported, largely in the form of theorems and their proofs, into an extensive list of appendices.

\section{SU(3) HALDANE-SHASTRY MODEL}

The SU(3) $1 / r^{2}$ or Haldane-Shastry model [19, 21] is most conveniently formulated by embedding the one-dimensional chain with periodic boundary conditions into the complex plane by mapping it onto the unit circle with the $\mathrm{SU}(3)$ spins located at complex positions $\eta_{\alpha}=\exp \left(i \frac{2 \pi}{N} \alpha\right)$, where $N$ denotes the number of sites and $\alpha=1, \ldots, N$. The Hamiltonian is given by

$$
H_{\mathrm{SU}(3)}=\left(\frac{2 \pi}{N}\right)^{2} \sum_{\alpha<\beta}^{N} \frac{\boldsymbol{J}_{\alpha} \cdot \boldsymbol{J}_{\beta}}{\left|\eta_{\alpha}-\eta_{\beta}\right|^{2}},
$$

where $\boldsymbol{J}_{\alpha}=\frac{1}{2} \sum_{\sigma \tau} c_{\alpha \sigma}^{\dagger} \boldsymbol{\lambda}_{\sigma \tau} c_{\alpha \tau}$ is the 8-dimensional SU(3) spin vector, $\boldsymbol{\lambda}$ a vector consisting of the eight Gell-Mann matrices (see App. $\mathrm{A}$ ), and $\sigma$ and $\tau$ are $\mathrm{SU}(3)$ spin or color indices, which take the values blue (b), red (r), or green (g). For all practical purposes, it is convenient to express $H_{\mathrm{SU}(3)}$ directly in terms of colorflip operators $e_{\alpha}^{\sigma \tau} \equiv c_{\alpha \sigma}^{\dagger} c_{\alpha \tau}$ :

$$
\begin{aligned}
H_{\mathrm{SU}(3)} & =\frac{2 \pi^{2}}{N^{2}} \sum_{\alpha<\beta}^{N} \sum_{\sigma \tau}^{3} \frac{e_{\alpha}^{\sigma \tau} e_{\beta}^{\tau \sigma}-\frac{1}{27}}{\left|\eta_{\alpha}-\eta_{\beta}\right|^{2}} \\
& =\frac{2 \pi^{2}}{N^{2}} \sum_{\alpha<\beta}^{N} \sum_{\sigma \tau}^{3} \frac{e_{\alpha}^{\sigma \tau} e_{\beta}^{\tau \sigma}}{\left|\eta_{\alpha}-\eta_{\beta}\right|^{2}}-\frac{\pi^{2}}{N^{2}} \frac{N\left(N^{2}-1\right)}{36}
\end{aligned}
$$

where the color sum includes terms with $\sigma=\tau$.

The model is fully integrable even for a finite number of sites; the algebra of infinitely 

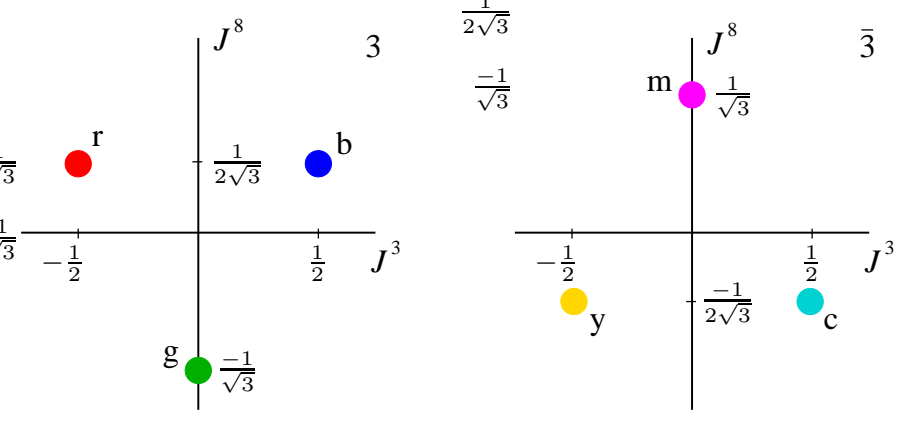

FIG. 1: (Color online) Weight diagrams of the $\mathrm{SU}(3)$ representations 3 and $\overline{3} . J^{3}$ and $J^{8}$ denote the diagonal generators [25].

many conserved quantities is generated by the total $\mathrm{SU}(3)$ spin

$$
\left[H_{\mathrm{SU}(3)}, \boldsymbol{J}\right]=0, \quad \boldsymbol{J}=\sum_{\alpha=1}^{N} \boldsymbol{J}_{\alpha}
$$

and rapidity operators

$$
\left[H_{\mathrm{SU}(3)}, \Lambda^{a}\right]=0, \quad \Lambda^{a}=\frac{1}{2} \sum_{\alpha \neq \beta}^{N} \frac{\eta_{\alpha}+\eta_{\beta}}{\eta_{\alpha}-\eta_{\beta}} f^{a b c} J_{\alpha}^{b} J_{\beta}^{c},
$$

where $a, b, c=1, \ldots, 8$ and $f^{a b c}$ are the structure constants of $\mathrm{SU}(3)$ defined through $\left[\lambda^{a}, \lambda^{b}\right]=2 f^{a b c} \lambda^{c}$. The total $\mathrm{SU}(3)$ spin and rapidity operators do not commute mutually,

$$
\left[J^{a}, \Lambda^{b}\right]=f^{a b c} \Lambda^{c}
$$

and generate the infinite dimensional Yangian algebra $Y\left(\mathrm{sl}_{3}\right)[18,26]$.

\section{GROUND STATE}

The ground state of $H_{\mathrm{SU}(3)}$ for $N=3 M$ ( $M$ integer) is most easily formulated by Gutzwiller projection of a filled band (or Slater determinant (SD) state) containing a total of $N \mathrm{SU}(3)$ particles obeying Fermi statistics (see Fig. 2a)

$$
\left|\Psi_{0}\right\rangle=P_{\mathrm{G}} \prod_{|q| \leq q_{\mathrm{F}}} c_{q \mathrm{~b}}^{\dagger} c_{q \mathrm{r}}^{\dagger} c_{q \mathrm{~g}}^{\dagger}|0\rangle \equiv P_{\mathrm{G}}\left|\Psi_{\mathrm{SD}}^{N}\right\rangle .
$$

The Gutzwiller projector

$$
P_{\mathrm{G}}=\prod_{\alpha=1}^{N}\left(n_{\alpha}-2\right)\left(n_{\alpha}-3\right)
$$



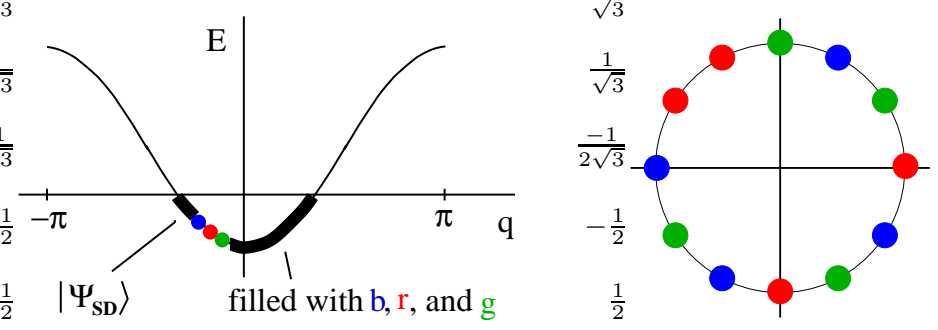

FIG. 2: (Color online) a) Total antisymmetric $N$-particle state. b) Typical configuration in $\left|\Psi_{0}\right\rangle$.

with $n_{\alpha} \equiv c_{\alpha \mathrm{b}}^{\dagger} c_{\alpha \mathrm{b}}+c_{\alpha \mathrm{r}}^{\dagger} c_{\alpha \mathrm{r}}+c_{\alpha \mathrm{g}}^{\dagger} c_{\alpha \mathrm{g}}$ eliminates configurations with more than one particle on any site, and, as the total number of particles equals the total number of sites, thereby effectively enforces single occupancy on all sites. As $\left|\Psi_{\mathrm{SD}}^{N}\right\rangle$ is an $\mathrm{SU}(3)$ singlet by construction and $P_{\mathrm{G}}$ commutes with $\mathrm{SU}(3)$ rotations, $\left|\Psi_{0}\right\rangle$ is an $\mathrm{SU}(3)$ singlet as well.

If one interprets the state $\left|0_{\mathrm{g}}\right\rangle \equiv \prod_{\alpha=1}^{N} c_{\alpha \mathrm{g}}^{\dagger}|0\rangle$ as a reference state and the colorflip operators $e^{\mathrm{bg}}$ and $e^{\mathrm{rg}}$ as "particle creation operators", the ground state (6) can be rewritten as $[20,21]$

$$
\left|\Psi_{0}\right\rangle=\sum_{\left\{z_{i}, w_{k}\right\}} \Psi_{0}\left[z_{i} ; w_{k}\right] e_{z_{1}}^{\mathrm{bg}} \ldots e_{z_{M}}^{\mathrm{bg}} e_{w_{1}}^{\mathrm{rg}} \ldots e_{w_{M}}^{\mathrm{rg}}\left|0_{\mathrm{g}}\right\rangle,
$$

where the sum extends over all possible ways to distribute the positions of the blue particles $z_{1}, \ldots, z_{M}$ and red particles $w_{1}, \ldots, w_{M}$ over the $N$ sites (see Fig. 20). The ground state wave function is given by

$$
\Psi_{0}\left[z_{i} ; w_{k}\right] \equiv \prod_{i<j}^{M_{1}}\left(z_{i}-z_{j}\right)^{2} \prod_{k<l}^{M_{2}}\left(w_{k}-w_{l}\right)^{2} \prod_{i=1}^{M_{1}} \prod_{k=1}^{M_{2}}\left(z_{i}-w_{k}\right) \prod_{i=1}^{M_{1}} z_{i} \prod_{k=1}^{M_{2}} w_{k}
$$

with $M_{1}=M_{2}=M$, as derived in App. B. The ground state energy is

$$
E_{0}=-\frac{\pi^{2}}{18}\left(N+\frac{7}{N}\right) \text {. }
$$

The total momentum, as defined through $e^{i p}=\Psi_{0}\left[\eta_{1} z_{i}, \eta_{1} w_{k}\right] / \Psi_{0}\left[z_{i}, w_{k}\right]$ with $\eta_{1}=\exp \left(i \frac{2 \pi}{N}\right)$, is $p=0$ regardless of $M$. (This is only true for $\mathrm{SU}(n)$ with $n$ odd.)

We will now prove by explicit calculation that (9) is an exact eigenstate of the Hamiltonian (2). Parts of this calculation will be used in Sec. IVB to prove the exactness of our proposed wave function for the coloron excitation.

To evaluate the action of $H_{\mathrm{SU}(3)}$ on (9) we first replace $e_{\alpha}^{\mathrm{gg}} e_{\beta}^{\mathrm{gg}}$ in (2) by $\left(1-e_{\alpha}^{\mathrm{bb}}-e_{\alpha}^{\mathrm{rr}}\right)(1-$ $\left.e_{\beta}^{\mathrm{bb}}-e_{\beta}^{\mathrm{rr}}\right)$,

$$
H_{\mathrm{SU}(3)}=\frac{2 \pi^{2}}{N^{2}} \sum_{\alpha \neq \beta}^{N} \frac{1}{\left|\eta_{\alpha}-\eta_{\beta}\right|^{2}}\left(e_{\alpha}^{\mathrm{bg}} e_{\beta}^{\mathrm{gb}}+e_{\alpha}^{\mathrm{rg}} e_{\beta}^{\mathrm{gr}}+e_{\alpha}^{\mathrm{br}} e_{\beta}^{\mathrm{rb}}\right)
$$




$$
\begin{aligned}
& +\frac{2 \pi^{2}}{N^{2}} \sum_{\alpha \neq \beta}^{N} \frac{1}{\left|\eta_{\alpha}-\eta_{\beta}\right|^{2}}\left(e_{\alpha}^{\mathrm{bb}} e_{\beta}^{\mathrm{bb}}+e_{\alpha}^{\mathrm{rr}} e_{\beta}^{\mathrm{rr}}+e_{\alpha}^{\mathrm{bb}} e_{\beta}^{\mathrm{rr}}\right) \\
& -\frac{2 \pi^{2}}{N^{2}} \sum_{\alpha \neq \beta}^{N} \frac{1}{\left|\eta_{\alpha}-\eta_{\beta}\right|^{2}}\left(e_{\alpha}^{\mathrm{bb}}+e_{\alpha}^{\mathrm{rr}}\right)+\frac{2}{3} \frac{\pi^{2}}{N^{2}} \sum_{\alpha \neq \beta}^{N} \frac{1}{\left|\eta_{\alpha}-\eta_{\beta}\right|^{2}},
\end{aligned}
$$

and then evaluate each term separately.

The first term $\left[e_{\alpha}^{\mathrm{bg}} e_{\beta}^{\mathrm{gb}} \Psi_{0}\right]\left[z_{i} ; w_{k}\right]$, which vanishes unless one of the $z_{i}$ 's is equal to $\eta_{\alpha}$, yields through Taylor expansion

$$
\begin{aligned}
{\left[\sum_{\alpha \neq \beta}^{N} \frac{e_{\alpha}^{\mathrm{bg}} e_{\beta}^{\mathrm{gb}}}{\left|\eta_{\alpha}-\eta_{\beta}\right|^{2}} \Psi_{0}\right]\left[z_{i} ; w_{k}\right]=} & \sum_{i=1}^{M_{1}} \sum_{\beta \neq i}^{N} \frac{\eta_{\beta}}{\left|z_{i}-\eta_{\beta}\right|^{2}} \frac{\Psi_{0}\left[\ldots, z_{i-1}, \eta_{\beta}, z_{i+1}, \ldots ; w_{k}\right]}{\eta_{\beta}} \\
= & \sum_{i=1}^{M_{1}} \sum_{m=0}^{N-1} \frac{A_{m} z_{i}^{m+1}}{m !} \frac{\partial^{m}}{\partial z_{i}^{m}} \frac{\Psi_{0}}{z_{i}} \\
= & \frac{M_{1}}{12}\left(N^{2}+8 M_{1}^{2}-6 M_{1}(N+1)+3\right) \Psi_{0} \\
& -\frac{N-3}{2} \sum_{i=1}^{M_{1}} \sum_{k=1}^{M_{2}} \frac{z_{i}}{z_{i}-w_{k}} \Psi_{0}+\sum_{i \neq j}^{M_{1}} \frac{z_{i}^{2}}{\left(z_{i}-z_{j}\right)^{2}} \Psi_{0} \\
& +2 \sum_{i \neq j}^{M_{1}} \sum_{k=1}^{M_{2}} \frac{z_{i}^{2}}{\left(z_{i}-z_{j}\right)\left(z_{i}-w_{k}\right)} \Psi_{0} \\
& +\frac{1}{2} \sum_{i=1}^{M_{1}} \sum_{k \neq l}^{M_{2}} \frac{z_{i}^{2}}{\left(z_{i}-w_{k}\right)\left(z_{i}-w_{l}\right)} \Psi_{0}
\end{aligned}
$$

where we have used $\operatorname{deg}_{z_{i}} \Psi_{0}\left[z_{i} ; w_{k}\right]=N-1$ and defined $A_{m} \equiv-\sum_{\alpha=1}^{N-1} \eta_{\alpha}^{2}\left(\eta_{\alpha}-1\right)^{m-2}$. Evaluation of the latter yields $A_{0}=(N-1)(N-5) / 12, A_{1}=-(N-3) / 2, A_{2}=1$, and $A_{m}=0$ for $2<m \leq N-1$ (see App. (C)). Furthermore, we have used

$$
\frac{x^{2}}{(x-y)(x-z)}+\frac{y^{2}}{(y-x)(y-z)}+\frac{z^{2}}{(z-x)(z-y)}=1, \quad x, y, z \in \mathbb{C} .
$$

The second term $\left[e_{\alpha}^{\mathrm{rg}} e_{\beta}^{\mathrm{gr}} \Psi_{0}\right]\left[z_{i} ; w_{k}\right]$ can be treated in the same way and yields together with the first term in (13)

$$
-\frac{N-3}{2} \sum_{i=1}^{M_{1}} \sum_{k=1}^{M_{2}} \frac{z_{i}}{z_{i}-w_{k}}+\frac{N-3}{2} \sum_{i=1}^{M_{1}} \sum_{k=1}^{M_{2}} \frac{w_{k}}{z_{i}-w_{k}}=-\frac{N-3}{2} M_{1} M_{2},
$$

and with one part of (14) and (15)

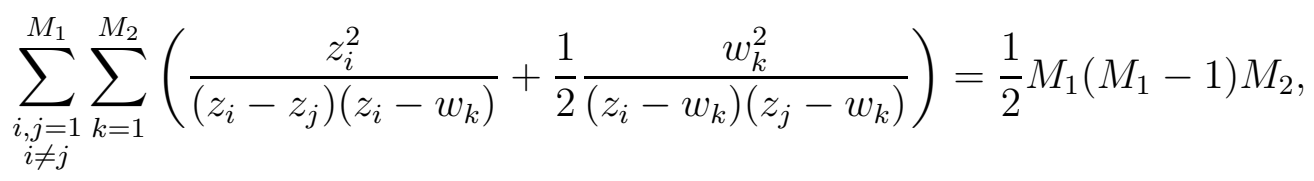


as well as similar expressions for $z_{i} \leftrightarrow w_{k}$.

For the third term of (11) we obtain

$$
\begin{aligned}
& {\left[\sum_{\alpha \neq \beta}^{N} \frac{e_{\alpha}^{\mathrm{br}} e_{\beta}^{\mathrm{rb}}}{\left|\eta_{\alpha}-\eta_{\beta}\right|^{2}} \Psi_{0}\right]\left[z_{i} ; w_{k}\right]=\sum_{i=1}^{M_{1}} \sum_{k=1}^{M_{2}} \frac{z_{i} w_{k}}{\left(z_{i}-w_{k}\right)^{2}} \prod_{j \neq i}^{M_{1}}\left(1+\frac{z_{i}-w_{k}}{z_{j}-z_{i}}\right) \prod_{l \neq k}^{M_{2}}\left(1-\frac{z_{i}-w_{k}}{w_{l}-w_{k}}\right) \Psi_{0} } \\
&= \sum_{i=1}^{M_{1}} \sum_{k=1}^{M_{2}} \frac{z_{i} w_{k}}{\left(z_{i}-w_{k}\right)^{2}} \Psi_{0} \\
&-\sum_{i \neq j}^{M_{1}} \sum_{k=1}^{M_{2}} \frac{z_{i} w_{k}}{\left(z_{i}-z_{j}\right)\left(z_{i}-w_{k}\right)} \Psi_{0}-\sum_{i=1}^{M_{1}} \sum_{k \neq l}^{M_{2}} \frac{z_{i} w_{k}}{\left(w_{k}-z_{i}\right)\left(w_{k}-w_{l}\right)} \Psi_{0} \\
&+\sum_{i=1}^{M_{1}} \sum_{k=1}^{M_{2}} \sum_{m=2}^{M_{1}-1} \frac{1}{m !} \sum_{\left\{a_{j}\right\}} \frac{z_{i} w_{k}\left(z_{i}-w_{k}\right)^{m-2}}{\left(z_{a_{1}}-z_{i}\right) \cdots\left(z_{a_{m}}-z_{i}\right)} \Psi_{0} \\
&+\sum_{i=1}^{M_{1}} \sum_{k=1}^{M_{2}} \sum_{n=2}^{M_{2}-1} \frac{(-1)^{n}}{n !} \sum_{\left\{b_{l}\right\}} \frac{z_{i} w_{k}\left(z_{i}-w_{k}\right)^{n-2}}{\left(w_{b_{1}}-w_{k}\right) \cdots\left(w_{b_{n}}-w_{k}\right)} \Psi_{0} \\
&+ \sum_{i=1}^{M_{1}} \sum_{k=1}^{M_{2}} \sum_{m=1}^{M_{1}-1} \sum_{n=1}^{M_{2}-1} \frac{(-1)^{n}}{m ! n !} \\
& \sum_{\left\{a_{j}\right\}\left\{b_{l}\right\}} \frac{z_{i} w_{k}\left(z_{i}-w_{k}\right)^{m+n-2}}{\left(z_{a_{1}}-z_{i}\right) \cdots\left(z_{a_{m}}-z_{i}\right)\left(w_{b_{1}}-w_{k}\right) \cdots\left(w_{b_{n}}-w_{k}\right)} \Psi_{0}
\end{aligned}
$$

where $\left\{a_{j}\right\}\left(\left\{b_{l}\right\}\right)$ is a set of integers between 1 and $M_{1}\left(M_{2}\right)$. The summations run over all possible ways to distribute the $z_{a_{j}}\left(w_{b_{l}}\right)$ over the blue (red) coordinates, where $z_{i}\left(w_{k}\right)$ is excluded. The two terms (19) and (20) vanish due to

Theorem 1 Let $M \geq 3, z \in \mathbb{C}$, and $z_{1}, \ldots, z_{M} \in \mathbb{C}$ distinct. Then,

$$
\sum_{i=1}^{M} \frac{z_{i}\left(z_{i}-z\right)^{M-3}}{\prod_{j \neq i}^{M}\left(z_{j}-z_{i}\right)}=0 .
$$

A proof of Theorem 1 is given in App. D. The last term (21) can be simplified using a theorem due to Ha and Haldane [21]:

Theorem 2 Let $\left\{a_{j}\right\}$ be a set of distinct integers between 1 and $M_{1}$, and $\left\{b_{l}\right\}$ a set of distinct integers between 1 and $M_{2}$. Then,

$$
\begin{gathered}
\sum_{i=1}^{M_{1}} \sum_{k=1}^{M_{2}} \sum_{m=1}^{M_{1}-1} \sum_{n=1}^{M_{2}-1} \sum_{\left\{a_{j}\right\}\left\{b_{l}\right\}} \frac{(-1)^{n}}{m ! n !} \frac{z_{i} w_{k}\left(z_{i}-w_{k}\right)^{m+n-2}}{\left(z_{a_{1}}-z_{i}\right) \cdots\left(z_{a_{n}}-z_{i}\right)\left(w_{b_{1}}-w_{k}\right) \cdots\left(w_{b_{m}}-w_{k}\right)} \\
=-\sum_{m=1}^{\min \left(M_{1}, M_{2}\right)}\left(M_{1}-m\right)\left(M_{2}-m\right) .
\end{gathered}
$$


Furthermore, the two terms in line (18), together with the remainder of (14) and the corresponding expression from the second term of the Hamiltonian, can be simplified to yield $M_{1} M_{2}\left(M_{1}+M_{2}-2\right) \Psi_{0} / 2$.

Finally, the diagonal terms of (111) cancel the remainder of (13) as well as (17) and yield the additional constant

$$
\frac{2 \pi^{2}}{N^{2}}\left[\frac{1}{2}\left(M_{1}\left(M_{1}-1\right)+M_{2}\left(M_{2}-1\right)\right)+\frac{N^{2}-1}{12}\left(\frac{N}{3}-M_{1}-M_{2}\right)\right] .
$$

When collecting all terms evaluated above and setting $M_{1}=M_{2}=N / 3$ we obtain the ground state energy (10).

\section{ONE-COLORON STATES}

\section{A. Trial wave functions}

We now turn to the heart of our analysis, the elementary and fractionally quantized excitations, which we call colorons. In principle, there are two possible, non-equivalent constructions for localized excitations starting from (6). We may either create a particle with color $\sigma$ on a chain with $N=3 M+1$ sites before Gutzwiller projection,

$$
\left|\Psi_{\gamma \sigma}^{\mathrm{c}}\right\rangle=P_{\mathrm{G}} c_{\gamma \sigma}^{\dagger}\left|\Psi_{\mathrm{SD}}^{N-1}\right\rangle
$$

or annihilate a particle with color $\sigma$ on a chain with $N=3 M-1$ :

$$
\left|\Psi_{\gamma \bar{\sigma}}^{\mathrm{a}}\right\rangle=P_{\mathrm{G}} c_{\gamma \sigma}\left|\Psi_{\mathrm{SD}}^{N+1}\right\rangle
$$

In both cases, $c_{\gamma \sigma}^{\dagger}$ or $c_{\gamma \sigma}$ creates an inhomogeneity in color and charge before projection. The projection once again enforces one particle per site and thereby removes the charge inhomogeneity, while it commutes with color and thereby preserves the color inhomogeneity. Consequently, the trial states $\left|\Psi_{\gamma \sigma}^{\mathrm{c}}\right\rangle$ and $\left|\Psi_{\gamma \bar{\sigma}}^{\mathrm{a}}\right\rangle$ describe localized "excitations" of color $\sigma$ or complementary color $\bar{\sigma}$, respectively, at lattice site $\eta_{\gamma}$. Since $H_{\mathrm{SU}(3)}$ is translationally invariant, we of course expect neither of them, but only momentum eigenstates constructed from them via

$$
\left|\Psi_{n}\right\rangle \equiv \frac{1}{N} \sum_{\gamma=1}^{N} e^{-i \frac{2 \pi}{N} \gamma n}\left|\Psi_{\gamma}\right\rangle=\frac{1}{N} \sum_{\gamma=1}^{N}\left(\bar{\eta}_{\gamma}\right)^{n}\left|\Psi_{\gamma}\right\rangle,
$$

where $n$ is a momentum quantum number, to be eigenstates. 
The important thing to realize now is that only (23) or (24), but not both, can describe a valid excitation. To see this, let us assume $\sigma=\mathrm{b}$ for ease in presentation, and note that (23) is apart from a normalization factor equivalent to

$$
\left|\Psi_{\gamma \mathrm{b}}^{\mathrm{c}}\right\rangle=P_{\mathrm{G}} c_{\gamma \mathrm{r}} c_{\gamma \mathrm{g}}\left|\Psi_{\mathrm{SD}}^{N+2}\right\rangle
$$

i.e., creation of a blue particle before projection is tantamount to annihilation of both a red and a green particle at site $\eta_{\gamma}$. If momentum eigenstates constructed from $\left|\Psi_{\gamma \bar{\sigma}}^{\mathrm{a}}\right\rangle$ via (25) were energy eigenstates, the anti-red (cyan) and anti-green (magenta) coloron excitations in (26) would individually seek to be momentum eigenstates, which implies that a trial wave function forcing them to sit on the same site would not be an energy eigenstate. The same argument can be made the other way round.

\section{B. One-coloron wave functions}

In the following, we show by explicit calculation that momentum eigenstates constructed

from $\left|\Psi_{\gamma \bar{\sigma}}^{\mathrm{a}}\right\rangle$ via (25) are exact eigenstates of $H_{\mathrm{SU}(3)}$. For simplicity, we choose $\bar{\sigma}=\overline{\mathrm{b}}=\mathrm{y}$ (an anti-blue or yellow coloron) and express $\left|\Psi_{\gamma \bar{b}}^{\mathrm{a}}\right\rangle$ through the corresponding wave function

$$
\Psi_{\gamma}\left[z_{i} ; w_{k}\right]=\prod_{i=1}^{M_{1}}\left(\eta_{\gamma}-z_{i}\right) \Psi_{0}\left[z_{i} ; w_{k}\right] \equiv \psi_{\gamma} \Psi_{0}
$$

with $\Psi_{0}$ given by (9) and $M_{1}=(N-2) / 3, M_{2}=(N+1) / 3$. The momentum eigenstate $\left|\Psi_{n \overline{\mathrm{b}}}^{\mathrm{a}}\right\rangle$ is then given by

$$
\Psi_{n}\left[z_{i} ; w_{k}\right]=\frac{1}{N} \sum_{\gamma=1}^{N}\left(\bar{\eta}_{\gamma}\right)^{n} \Psi_{\gamma}\left[z_{i} ; w_{k}\right]
$$

(where $n$ is shifted with respect to $n$ in (25) by a constant depending on which momenta are occupied in the Slater determinant state). The momentum of (28) is

$$
p=\frac{4 \pi}{3}-\frac{2 \pi}{N}\left(n+\frac{1}{3}\right), \quad 0 \leq n \leq M_{1}
$$

The energy is given by

$$
\begin{aligned}
E_{n} & =-\frac{\pi^{2}}{18}\left(N+\frac{1}{N}+\frac{2}{N^{2}}\right)+\frac{3 \pi^{2}}{N^{2}}\left(M_{1}-n\right) n \\
& =E_{0}+\frac{2}{9} \frac{\pi^{2}}{N^{2}}+\epsilon(p),
\end{aligned}
$$




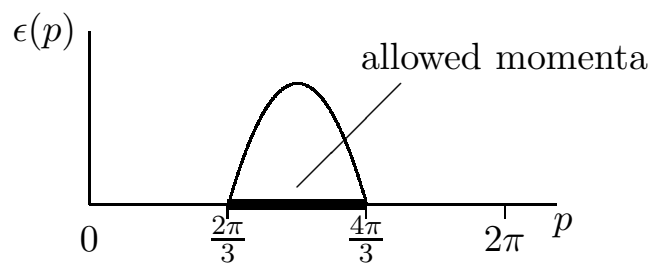

FIG. 3: One-coloron dispersion relation.

where we have defined the one-coloron dispersion relation (see Fig. 3)

$$
\epsilon(p)=\frac{3}{4}\left(\frac{\pi^{2}}{9}-(p-\pi)^{2}\right)
$$

For $M_{1}<n<N, \Psi_{n}$ vanishes identically.

To prove that $\Psi_{n}$ represents an energy eigenstate, note that all terms of the Hamiltonian (111) yield the same expressions as for the wave function $\Psi_{0}$ except those terms containing $e_{\alpha}^{\mathrm{bg}} e_{\beta}^{\mathrm{gb}}$ and $e_{\alpha}^{\mathrm{br}} e_{\beta}^{\mathrm{rb}}$. Hence, it is sufficient to investigate those further.

To begin with, the polynomial $\psi_{\gamma}$ defined in (27) can be written as $\sum_{m=0}^{M_{1}}(-1)^{M_{1}-m} \eta_{\gamma}^{m} \mathcal{S}^{M_{1}}\left(z_{1} \cdots z_{M_{1}-m}\right)$, where $\mathcal{S}^{M}\left(z_{1} \cdots z_{q}\right)$ is the sum over all possible ways to choose $q$ coordinates out of $z_{1}, \ldots, z_{M}$, e.g. $\mathcal{S}^{3}\left(z_{1} z_{2}\right)=z_{1} z_{2}+z_{1} z_{3}+z_{2} z_{3}$. Hence, we find in addition to (12)-(15) the two terms (note that $\operatorname{deg}_{z_{i}} \Psi_{\gamma}=\operatorname{deg}_{w_{k}} \Psi_{\gamma}=N-1$ )

$$
\Psi_{0} \sum_{i=1}^{M_{1}}\left(\frac{1}{2} z_{i}^{2} \frac{\partial^{2}}{\partial z_{i}^{2}}+\sum_{j \neq i}^{M_{1}} \frac{2 z_{i}^{2}}{z_{i}-z_{j}} \frac{\partial}{\partial z_{i}}-\frac{N-3}{2} z_{i} \frac{\partial}{\partial z_{i}}\right) \psi_{\gamma}+\Psi_{0} \sum_{i=1}^{M_{1}} \sum_{k=1}^{M_{2}} \frac{z_{i}^{2}}{z_{i}-w_{k}} \frac{\partial}{\partial z_{i}} \psi_{\gamma}
$$

Fourier transformation (28) of these yields

$$
\left[M_{1}\left(M_{1}-\frac{N-1}{2}\right)-n\left(n-\frac{N-1}{2}\right)\right] \Psi_{n}-\frac{1}{N} \Psi_{0} \sum_{\gamma=1}^{N} \sum_{i=1}^{M_{1}} \sum_{k=1}^{M_{2}} \frac{z_{i}^{2}\left(\bar{\eta}_{\gamma}\right)^{n}}{\left(z_{i}-w_{k}\right)\left(\eta_{\gamma}-z_{i}\right)} \psi_{\gamma} .
$$

Furthermore,

$$
\begin{aligned}
{\left[\sum_{\alpha \neq \beta}^{N} \frac{e_{\alpha}^{\mathrm{br}} e_{\beta}^{\mathrm{rb}}}{\left|\eta_{\alpha}-\eta_{\beta}\right|^{2}} \Psi_{n}\right]\left[z_{i} ; w_{k}\right]=\sum_{i=1}^{M_{1}} \sum_{k=1}^{M_{2}} \frac{z_{i} w_{k}}{\left(z_{i}-w_{k}\right)^{2}} \prod_{j \neq i}^{M_{1}} \frac{z_{j}-w_{k}}{z_{j}-z_{i}} \prod_{l \neq k}^{M_{2}} \frac{w_{l}-z_{i}}{w_{l}-w_{k}} \Psi_{n} } \\
+\frac{\Psi_{0}}{N} \sum_{\gamma=1}^{N} \sum_{i=1}^{M_{1}} \sum_{k=1}^{M_{2}} \frac{z_{i} w_{k}\left(\bar{\eta}_{\gamma}\right)^{n}}{\left(z_{i}-w_{k}\right)\left(\eta_{\gamma}-z_{i}\right)} \prod_{j \neq i}^{M_{1}} \frac{z_{j}-w_{k}}{z_{j}-z_{i}} \prod_{l \neq k}^{M_{2}} \frac{w_{l}-z_{i}}{w_{l}-w_{k}} \psi_{\gamma} .
\end{aligned}
$$

The term on the right of (34) is identical to a corresponding term in the ground state calculation, whereas (35) taken together with the second term in (33) gives

$$
\left[-\frac{1}{2} n(n+1)+\frac{1}{2} M_{1}\left(M_{1}+1\right)\right] \Psi_{n}
$$

where we have used 
Theorem 3 For $M_{1}, M_{2} \in \mathbb{N}, M_{1} \leq M_{2}, z_{1}, \ldots, z_{M_{1}} \in \mathbb{C}$ distinct, $w_{1}, \ldots, w_{M_{2}} \in \mathbb{C}$ distinct, and $0 \leq n \leq M_{1}$ the following is valid:

$$
\begin{gathered}
\sum_{i=1}^{M_{1}} \sum_{k=1}^{M_{2}} \frac{z_{i}}{z_{i}-w_{k}}\left[w_{k} \prod_{j \neq i}^{M_{1}} \frac{z_{j}-w_{k}}{z_{j}-z_{i}} \prod_{l \neq k}^{M_{2}} \frac{w_{l}-z_{i}}{w_{l}-w_{k}}-z_{i}\right] \sum_{\gamma=1}^{N}\left(\bar{\eta}_{\gamma}\right)^{n} \prod_{j \neq i}^{M_{1}}\left(\eta_{\gamma}-z_{j}\right) \\
=\left[-\frac{1}{2} n(n+1)+\frac{1}{2} M_{1}\left(M_{1}+1\right)\right] \sum_{\gamma=1}^{N}\left(\bar{\eta}_{\gamma}\right)^{n} \prod_{i=1}^{M_{1}}\left(\eta_{\gamma}-z_{i}\right) .
\end{gathered}
$$

The proof of Theorem 3 is given in App. E. Collecting the terms obtained above, we find that $\Psi_{n}$ is an exact eigenstate of the Hamiltonian (11) with energy (30).

\section{Quantum numbers of colorons}

In the preceding section we have shown that the elementary coloron excitations are constructed by annihilation of a particle of color $\sigma$ from an overall color singlet $\left|\Psi_{\mathrm{SD}}^{N+1}\right\rangle$ before Gutzwiller projection. Since they are hole-like excitations, they transform according to the representation $\overline{3}$ conjugate to the fundamental representation 3 of the original particles on the sites of the chain. This can also be seen by acting with the total SU(3) spin generators (3) on the wave function (27). Our result agrees with results obtained for the spectrum of the SU(3) 1 Wess-Zumino-Witten (WZW) model by Bouwknegt and Schoutens 27, 28].

It is straightforward to read off the quantum or exclusion statistics [23, 24] of colorpolarized colorons (i.e., colorons of the same color). Consider a chain with $N=3 M-1$ sites and a single yellow coloron. According to (6), (24), and (25), there are as many single particle orbitals available to the coloron as there are blue particles in the Slater determinant state, that is, $M$. If we now were to create three additional yellow colorons, the Slater determinant state would have to contain three more particles, one of each color. This implies there would be one additional orbital, while the three additional colorons would occupy three orbitals, meaning that the number of orbitals available for our original coloron would be reduced by two. The statistical parameter is hence given by $g=2 / 3$. The fractional statistics manifests

itself further in the exponents of the algebraic decay of the form factor of the dynamical structure factor [29, 30] as well as in the thermodynamics of the model [31, 32]. A similar exclusion statistics exists in the conformal field theory spectrum of WZW models [28, 33]. The exclusion statistics among colorons of different colors as well as the state counting for 
$\mathrm{SU}(n)(n \geq 3)$ spin chains in general is highly non-trivial and will be subject of a future publication [34].

\section{TWO-COLORON STATES}

For $N=3 M-2$, there are at least two colorons present. Two yellow colorons localized at lattice sites $\eta_{\gamma}$ and $\eta_{\delta}$ are described by the wave function

$$
\Psi_{\gamma \delta}\left[z_{i} ; w_{k}\right]=\prod_{i=1}^{M_{1}}\left(\eta_{\gamma}-z_{i}\right)\left(\eta_{\delta}-z_{i}\right) \Psi_{0}\left[z_{i} ; w_{k}\right] \equiv \psi_{\gamma \delta} \Psi_{0},
$$

where $M_{1}=(N-4) / 3$ and $M_{2}=(N+2) / 3$. Momentum eigenstates are once again constructed by Fourier transformation

$$
\Psi_{m n}\left[z_{i} ; w_{k}\right]=\frac{1}{N^{2}} \sum_{\gamma \delta}^{N}\left(\bar{\eta}_{\gamma}\right)^{m}\left(\bar{\eta}_{\delta}\right)^{n} \Psi_{\gamma \delta}\left[z_{i} ; w_{k}\right], \quad 0 \leq n \leq m \leq M_{1} .
$$

In analogy to the SU(2) HSM we neither expect these states for fixed $m$ and $n$ to be energy eigenstates, nor states with different sets of quantum numbers $(m, n)$ with the same $m+n$ (and hence the same total momentum) to be orthogonal to each other. Energy eigenstates, however, can be constructed as follows.

As for the one-coloron states, application of the Hamiltonian generates two contributions in addition to those familiar from the the ground state calculation. First, for the terms containing $e_{\alpha}^{\mathrm{bg}} e_{\beta}^{\mathrm{gb}}$ we obtain (32) with $\psi_{\gamma}$ replaced by $\psi_{\gamma \delta}$. The first term in the resulting expression can be treated in analogy to the two-spinon states in the SU(2) HSM [35]. Specifically, we find

$$
\begin{aligned}
& \sum_{i=1}^{M_{1}}\left(\frac{1}{2} z_{i}^{2} \frac{\partial^{2}}{\partial z_{i}^{2}}+\sum_{j \neq i}^{M_{1}} \frac{2 z_{i}^{2}}{z_{i}-z_{j}} \frac{\partial}{\partial z_{i}}-\frac{N-3}{2} z_{i} \frac{\partial}{\partial z_{i}}\right) \psi_{\gamma \delta}=M_{1}\left(2 M_{1}-N+2\right) \psi_{\gamma \delta} \\
& +\left[-\eta_{\gamma}^{2} \frac{\partial^{2}}{\partial \eta_{\gamma}^{2}}-\eta_{\delta}^{2} \frac{\partial^{2}}{\partial \eta_{\delta}^{2}}+\frac{N-3}{2}\left(\eta_{\gamma} \frac{\partial}{\partial \eta_{\gamma}}+\eta_{\delta} \frac{\partial}{\partial \eta_{\delta}}\right)\right] \psi_{\gamma \delta}-\frac{1}{\eta_{\gamma}-\eta_{\delta}}\left[\eta_{\gamma}^{2} \frac{\partial}{\partial \eta_{\gamma}}-\eta_{\delta}^{2} \frac{\partial}{\partial \eta_{\delta}}\right] \psi_{\gamma \delta}
\end{aligned}
$$

where we have used (16) three times. Applying this identity to the momentum eigenstates (38), we obtain

$$
\begin{aligned}
-M_{1}\left(M_{1}+2\right) \Psi_{m n}+ & {\left[\left(\frac{3}{2} M_{1}+1-m\right) m+\left(\frac{3}{2} M_{1}+1-n\right) n-\frac{m-n}{2}\right] \Psi_{m n} } \\
& -\sum_{\ell=1}^{\ell_{m}}(m-n+2 \ell) \Psi_{m+\ell, n-\ell},
\end{aligned}
$$


where $\ell_{\mathrm{m}}=\min \left(M_{1}-m, n\right)$, and we have used

$$
\frac{x+y}{x-y}\left(x^{m} y^{n}-x^{n} y^{m}\right)=2 \sum_{\ell=0}^{m-n} x^{m-\ell} y^{n+\ell}-\left(x^{m} y^{n}+x^{n} y^{m}\right), \quad m \geq n .
$$

The second term of (32) applied to the states $\Psi_{m n}$ yields

$$
-\frac{1}{N^{2}} \Psi_{0} \sum_{\gamma \delta}^{N} \sum_{i=1}^{M_{1}} \sum_{k=1}^{M_{2}} \frac{z_{i}^{2}\left(\bar{\eta}_{\gamma}\right)^{m}\left(\bar{\eta}_{\delta}\right)^{n}}{z_{i}-w_{k}}\left(\frac{1}{\eta_{\gamma}-z_{i}}+\frac{1}{\eta_{\delta}-z_{i}}\right) \psi_{\gamma \delta}
$$

The second contribution not familiar from the ground state calculation is given by terms arising in

$$
\begin{aligned}
{\left[\sum_{\alpha \neq \beta}^{N} \frac{e_{\alpha}^{\mathrm{br}} e_{\beta}^{\mathrm{rb}}}{\left|\eta_{\alpha}-\eta_{\beta}\right|^{2}} \Psi_{m n}\right]\left[z_{i} ; w_{k}\right]=\sum_{i=1}^{M_{1}} \sum_{k=1}^{M_{2}} \frac{z_{i} w_{k}}{\left(z_{i}-w_{k}\right)^{2}} \prod_{j \neq i}^{M_{1}} \frac{z_{j}-w_{k}}{z_{j}-z_{i}} \prod_{l \neq k}^{M_{2}} \frac{w_{l}-z_{i}}{w_{l}-w_{k}} \Psi_{m n} } \\
+\frac{\Psi_{0}}{N^{2}} \sum_{\gamma \delta}^{N} \sum_{i=1}^{M_{1}} \sum_{k=1}^{M_{2}} \frac{z_{i} w_{k}\left(\bar{\eta}_{\gamma}\right)^{m}\left(\bar{\eta}_{\delta}\right)^{n}}{\left(z_{i}-w_{k}\right)\left(\eta_{\gamma}-z_{i}\right)} \prod_{j \neq i}^{M_{1}} \frac{z_{j}-w_{k}}{z_{j}-z_{i}} \prod_{l \neq k}^{M_{2}} \frac{w_{l}-z_{i}}{w_{l}-w_{k}} \psi_{\gamma \delta} \\
+\frac{\Psi_{0}}{N^{2}} \sum_{\gamma \delta}^{N} \sum_{i=1}^{M_{1}} \sum_{k=1}^{M_{2}} \frac{z_{i} w_{k}\left(\bar{\eta}_{\gamma}\right)^{m}\left(\bar{\eta}_{\delta}\right)^{n}}{\left(z_{i}-w_{k}\right)\left(\eta_{\delta}-z_{i}\right)} \prod_{j \neq i}^{M_{1}} \frac{z_{j}-w_{k}}{z_{j}-z_{i}} \prod_{l \neq k}^{M_{2}} \frac{w_{l}-z_{i}}{w_{l}-w_{k}} \psi_{\gamma \delta} \\
+\frac{\Psi_{0}}{N^{2}} \sum_{\gamma \delta}^{N} \sum_{i=1}^{M_{1}} \sum_{k=1}^{M_{2}} \frac{z_{i} w_{k}\left(\bar{\eta}_{\gamma}\right)^{m}\left(\bar{\eta}_{\delta}\right)^{n}}{\left(\eta_{\gamma}-z_{i}\right)\left(\eta_{\delta}-z_{i}\right)} \prod_{j \neq i}^{M_{1}} \frac{z_{j}-w_{k}}{z_{j}-z_{i}} \prod_{l \neq k}^{M_{2}} \frac{w_{l}-z_{i}}{w_{l}-w_{k}} \psi_{\gamma \delta} .
\end{aligned}
$$

The first term (41) is identical to the ground state calculation. The two following terms (42) and (43) can be combined together with (40) and Theorem 3 to yield

$$
\left[M_{1}\left(M_{1}+1\right)-\frac{1}{2} m(m+1)-\frac{1}{2} n(n+1)\right] \Psi_{m n} .
$$

The last term (44) can be simplified using

Theorem 4 For $M_{1}, M_{2} \in \mathbb{N}, M_{1} \leq M_{2}-1, z_{1}, \ldots, z_{M_{1}} \in \mathbb{C}$ distinct, $w_{1}, \ldots, w_{M_{2}} \in \mathbb{C}$ distinct, and $0 \leq n \leq m \leq M_{1}$, and $\ell_{\mathrm{m}}=\min \left(M_{1}-m, n\right)$ the following is valid:

$$
\begin{gathered}
\sum_{i=1}^{M_{1}} \sum_{k=1}^{M_{2}} z_{i} w_{k} \prod_{j \neq i}^{M_{1}} \frac{z_{j}-w_{k}}{z_{j}-z_{i}} \prod_{l \neq k}^{M_{2}} \frac{w_{l}-z_{i}}{w_{l}-w_{k}} \sum_{\gamma \delta}^{N}\left(\bar{\eta}_{\gamma}\right)^{m}\left(\bar{\eta}_{\delta}\right)^{n} \prod_{j \neq i}^{M_{1}}\left(\eta_{\gamma}-z_{j}\right)\left(\eta_{\delta}-z_{j}\right) \\
=\left(M_{1}-m\right) \sum_{\gamma \delta}^{N}\left(\bar{\eta}_{\gamma}\right)^{m}\left(\bar{\eta}_{\delta}\right)^{n} \prod_{i=1}^{M_{1}}\left(\eta_{\gamma}-z_{i}\right)\left(\eta_{\delta}-z_{i}\right) \\
\quad-\sum_{\ell=1}^{\ell_{\mathrm{m}}}(m-n+2 \ell) \sum_{\gamma \delta}^{N}\left(\bar{\eta}_{\gamma}\right)^{m+\ell}\left(\bar{\eta}_{\delta}\right)^{n-\ell} \prod_{i=1}^{M_{1}}\left(\eta_{\gamma}-z_{i}\right)\left(\eta_{\delta}-z_{i}\right) .
\end{gathered}
$$


A proof of Theorem 4 is given in App. F

After collecting all terms, we obtain for the action of the Hamiltonian on the momentum eigenstates (38):

$$
\begin{aligned}
H_{\mathrm{SU}(3)}\left|\Psi_{m n}\right\rangle= & -\frac{\pi^{2}}{18}\left(N-\frac{17}{N}+\frac{52}{N^{2}}\right)\left|\Psi_{m n}\right\rangle \\
& +\frac{3 \pi^{2}}{N^{2}}\left[\left(M_{1}-m\right) m+\left(M_{1}-n\right) n-\frac{2}{3}(m-n)\right]\left|\Psi_{m n}\right\rangle \\
& -\frac{4 \pi^{2}}{N^{2}} \sum_{\ell=1}^{\ell_{\mathrm{m}}}(m-n+2 \ell)\left|\Psi_{m+\ell, n-\ell}\right\rangle .
\end{aligned}
$$

This Sutherland-type equation [36, 37] shows first of all that when we act with the Hamiltonian on the non-orthogonal basis state (38) with $m=m_{0}, n=n_{0}, m_{0} \geq n_{0}$, we only obtain terms (38) with $m \geq m_{0}$ and $n \leq n_{0}$ with fixed $m+n=m_{0}+n_{0}$, but no terms with $m<m_{0}$ or $n>n_{0}$. Consequently, states with $m=M_{1}$ or $n=0$ (or both) are exact eigenstates. Furthermore, we can obtain all the other exact eigenstates at each fixed $m+n$ (or fixed total momentum) by subsequently constructing an orthogonal basis of states starting from these exact eigenstates. (We have shown previously [38] that this method can be used to obtain all the spin polarized two spinon eigenstates of the SU(2) HSM.)

With all the numerical constants in place, however, (47) provides much more information than the direction of the scattering. We can solve for the energy eigenstates directly through the Ansatz

$$
\Phi_{m n}=\sum_{\ell=0}^{\ell_{\mathrm{m}}} a_{\ell}^{m n} \Psi_{m+\ell, n-\ell}
$$

Requiring

$$
H_{\mathrm{SU}(3)}\left|\Phi_{m n}\right\rangle=E_{m n}\left|\Phi_{m n}\right\rangle
$$

then yields the recursion relation

$$
a_{\ell}^{m n}=\frac{\left(\ell-\frac{5}{3}\right)(m-n+\ell-1)(m-n+2 \ell)}{\ell\left(m-n+\ell+\frac{2}{3}\right)(m-n+2 \ell-2)} a_{\ell-1}^{m n}, \quad a_{0}^{m n}=1,
$$

$\left(a_{1}^{m m}=-4 / 5\right)$ for the coefficients $a_{\ell}^{m n}$ as well as

$$
E_{m n}=-\frac{\pi^{2}}{18}\left(N-\frac{17}{N}+\frac{52}{N^{2}}\right)+\frac{3 \pi^{2}}{N^{2}}\left[\left(M_{1}-m\right) m+\left(M_{1}-n\right) n-\frac{2}{3}(m-n)\right] .
$$

for the two-coloron energies.

As we introduce single-coloron momenta according to

$$
p_{m}=\frac{4}{3} \pi-\frac{2 \pi}{N}(m+1), \quad p_{n}=\frac{4}{3} \pi-\frac{2 \pi}{N}\left(n+\frac{1}{3}\right),
$$


the energy (51) simplifies to

$$
E_{m n}=E_{0}+\frac{4}{9} \frac{\pi^{2}}{N^{2}}+\epsilon\left(p_{m}\right)+\epsilon\left(p_{n}\right)
$$

with $E_{0}$ given by (10) and the single-coloron dispersion relation $\epsilon(p)$ given by (31). The interpretation of (52) and (53) is as follows. First, the coloron excitations in the SU(3) HSM are (like the spinon excitations in the $\mathrm{SU}(2)$ model [38]) free, and the total energy is simply given by the sum of the kinetic energies of the individual colorons. This conclusion is consistent with the asymptotic Bethe Ansatz calculation by Essler [39], who has shown that the coloron-coloron scattering matrix is trivial, i.e., a momentum independent phase. Second, the difference in the individual coloron momenta (52) is quantized as

$$
p_{n}-p_{m}=\frac{2 \pi}{N}\left(\frac{2}{3}+\text { integer }\right),
$$

i.e., the minimal momentum spacing between color-polarized colorons is $2 / 3$ of the momentum spacing for fermions on a ring, $2 \pi / N$. We interpret this result as a manifestation of the fractional statistics of the colorons. This result appears to indicate that the spacing for particles with exclusion statistics $g$ is given by

$$
p_{n}-p_{m}=\frac{2 \pi}{N}(g+\text { integer }) .
$$

This identification is obviously consistent with the familiar cases of bosons and fermions, and may even constitute the most direct manifestation of Haldane's [24] fractional exclusion principle per se.

The fractional momenta (52) may also be interpreted in terms of an effective change in the periodic boundary conditions (PBCs). The allowed values for the total momenta of the two-coloron states are those for conventional PBCs,

$$
p_{n}+p_{m}=\frac{2 \pi}{N} \cdot \text { integer }
$$

where we have used the fact that $N+2$ in (52) is divisible by three. This is also required, as the wave function must come back to itself under a full translation around the ring. The allowed values for the difference in the momenta (154), by contrast, are those of a ring threaded by a magnetic flux $2 \pi \cdot 2 / 3$. In a certain sense, we may interpret this flux as a phase acquired by the wave function as one coloron goes "through" the other. There are, however, several subtleties associated with this interpretation [40]. 


\section{SU(3) SPIN CURRENTS}

In this section, we calculate the $\mathrm{SU}(3)$ spin currents, i.e., the eigenvalues of the diagonal rapidity operators $\Lambda^{3}$ and $\Lambda^{8}$. To this end, we first rewrite them in terms of colorflip operators:

$$
\begin{aligned}
& \Lambda^{3}=-\frac{1}{2} \sum_{\alpha \neq \beta}^{N} \frac{\eta_{\alpha}+\eta_{\beta}}{\eta_{\alpha}-\eta_{\beta}}\left(e_{\alpha}^{\mathrm{br}} e_{\beta}^{\mathrm{rb}}+\frac{1}{2} e_{\alpha}^{\mathrm{bg}} e_{\beta}^{\mathrm{gb}}-\frac{1}{2} e_{\alpha}^{\mathrm{rg}} e_{\beta}^{\mathrm{gr}}\right), \\
& \Lambda^{8}=-\frac{\sqrt{3}}{4} \sum_{\alpha \neq \beta}^{N} \frac{\eta_{\alpha}+\eta_{\beta}}{\eta_{\alpha}-\eta_{\beta}}\left(e_{\alpha}^{\mathrm{bg}} e_{\beta}^{\mathrm{gb}}+e_{\alpha}^{\mathrm{rg}} e_{\beta}^{\mathrm{gr}}\right) .
\end{aligned}
$$

The eigenvalue of $\Lambda^{8}$ is easier to evaluate. Using the Taylor expansion technique we obtain

$$
\begin{aligned}
{\left[\Lambda^{8} \Psi\right]\left[z_{i} ; w_{k}\right] } & =\frac{\sqrt{3}}{4} \sum_{m=0}^{N-2} \frac{B_{m}}{m !}\left[\sum_{i=1}^{M_{1}} z_{i}^{m+1} \frac{\partial^{m}}{\partial z_{i}^{m}} \frac{\Psi}{z_{i}}+\sum_{k=1}^{M_{2}} w_{k}^{m+1} \frac{\partial^{m}}{\partial w_{k}^{m}} \frac{\Psi}{w_{k}}\right] \\
& =\frac{\sqrt{3}}{4}(N-2)\left(M_{1}+M_{2}\right) \Psi-\frac{\sqrt{3}}{2}\left[\sum_{i=1}^{M_{1}} z_{i}^{2} \frac{\partial}{\partial z_{i}} \frac{\Psi}{z_{i}}+\sum_{k=1}^{M_{2}} w_{k}^{2} \frac{\partial}{\partial w_{k}} \frac{\Psi}{w_{k}}\right]
\end{aligned}
$$

where the wave function has to satisfy $\operatorname{deg}_{z_{i}} \Psi \leq N-1$ and $\operatorname{deg}_{w_{k}} \Psi \leq N-1$, and we have

defined $B_{m} \equiv \sum_{\alpha=1}^{N-1} \eta_{\alpha}\left(\eta_{\alpha}+1\right)\left(\eta_{\alpha}-1\right)^{m-1}$. Evaluation of the latter yields $B_{0}=N-2$, $B_{1}=-2$, and $B_{m}=0$ for $2 \leq m \leq N-2$ (see App. C).

With (59) we find for the ground state, the one-coloron states (28), and the two-coloron states (48)

$$
\begin{aligned}
\Lambda^{8}\left|\Psi_{0}\right\rangle & =0 \\
\Lambda^{8}\left|\Psi_{n}\right\rangle & =-\frac{\sqrt{3}}{4}\left(\frac{N-2}{3}-2 n\right)\left|\Psi_{n}\right\rangle, \\
\Lambda^{8}\left|\Phi_{m n}\right\rangle & =-\frac{\sqrt{3}}{4}\left(\frac{2 N-8}{3}-2 m-2 n\right)\left|\Phi_{m n}\right\rangle .
\end{aligned}
$$

The eigenvalues of $\Lambda^{3}$ can be obtained either by explicit calculation or be determined as follows. The fundamental representations of the Yangian $Y\left(\mathrm{sl}_{3}\right)$ can be constructed [26] from the representations of $\mathrm{sl}_{3}$ (i.e., $\mathrm{SU}(3)$ ) by the pull-back under the so-called evaluation homomorphism from $Y\left(\mathrm{sl}_{3}\right)$ into the universal enveloping algebra $U\left(\mathrm{sl}_{3}\right)$ of $\mathrm{sl}_{3}$. Explicitly, the ground state transforms according to the pull-back of the $\mathrm{SU}(3)$ singlet representation under $Y\left(\mathrm{sl}_{3}\right)$ transformations, the one-coloron states transform according to the pull-back of the representation $\overline{3}$, and the two-coloron states investigated in Sec. $\mathbb{V}$ according to the 

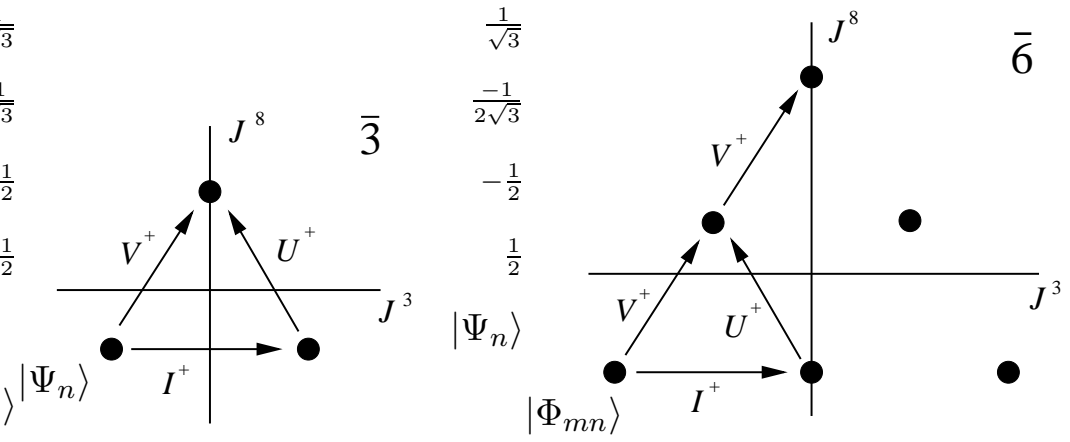

FIG. 4: Weight diagrams of the SU(3) representations $\overline{3}$ and $\overline{6}$.

pull-back of the representation $\overline{6}$ (as the two colorons are coupled symmetrically). For the fundamental representations of $Y\left(\mathrm{sl}_{3}\right)$ constructed in this way the ratio of the eigenvalues of $\Lambda^{3}$ and $\Lambda^{8}$ is equal to the ratio of the eigenvalues of $J^{3}$ and $J^{8}$. Hence, as the states $\left|\Psi_{n}\right\rangle$ and $\left|\Phi_{m n}\right\rangle$ are $\mathrm{SU}(3)$ lowest weight states (i.e., they are annihilated by $I^{-}=J^{1}-i J^{2}$, $V^{-}=J^{4}-i J^{5}$, and $U^{-}=J^{6}-i J^{7}$; see Fig. (4) , the eigenvalues of $\Lambda^{3}$ are simply $\sqrt{3}$ times the eigenvalues of $\Lambda^{8}$ given in (601)-(62).

\section{ASYMPTOTIC BETHE ANSATZ}

Let us now briefly compare our results with conclusions drawn from the asymptotic Bethe Ansatz (ABA). We wish to stress that the ABA is not an exact method. In the derivation of the $\mathrm{ABA}$ equations one assumes the existence of an asymptotic region in which the particles (or colorflips) are non-interacting [19]; an assumption which is not valid rigorously. In particular, the $\mathrm{ABA}$ equations have no direct connection to the exact eigenstates of the Hamiltonian [39] as it is the case for the standard Bethe Ansatz. It turns out, nevertheless, that the ABA reproduces the spectrum of the model, hence there is an indirect relation between solutions of the ABA equations and energy eigenstates. Let us now set up the formalism. The eigenstates of the SU(3) HSM are specified by two sets of pseudomomenta $k_{i}^{1}$ and $k_{i}^{2}$ satisfying the ABA equations [19, 22]

$$
\begin{aligned}
& N k_{i}^{1}=2 \pi I_{i}^{1}-\pi \sum_{j=1}^{M^{2}} \operatorname{sgn}\left(k_{i}^{1}-k_{j}^{2}\right)+\pi \sum_{j=1}^{M^{1}} \operatorname{sgn}\left(k_{i}^{1}-k_{j}^{1}\right), \\
& \pi \sum_{j=1}^{M^{1}} \operatorname{sgn}\left(k_{i}^{2}-k_{j}^{1}\right)=2 \pi I_{i}^{2}+\pi \sum_{j=1}^{M^{2}} \operatorname{sgn}\left(k_{i}^{2}-k_{j}^{2}\right),
\end{aligned}
$$


with two sets of mutually distinct integer (or half-integer) quantum numbers in the range

$$
\left|I_{i}^{1}\right| \leq \frac{1}{2}\left(N+M^{2}-M^{1}-1\right), \quad\left|I_{i}^{2}\right| \leq \frac{1}{2}\left(M^{1}-M^{2}-1\right) .
$$

In (63) we have restricted ourselves to 1-strings, i.e., the $k_{i}^{1,2}$ 's are real numbers, which means that we can only describe color-polarized states (for the general case see [22, 39]). The energy and momentum depend only on the first set of pseudomomenta,

$$
\begin{aligned}
E & =\frac{1}{4} \sum_{i=1}^{M^{1}}\left(\left(k_{i}^{1}\right)^{2}-\pi^{2}\right)+\frac{\pi^{2}}{N^{2}} \frac{N\left(N^{2}-1\right)}{18} \\
p & =\sum_{i=1}^{M^{1}}\left(k_{i}^{1}+\pi\right) \bmod 2 \pi
\end{aligned}
$$

where $k_{i}^{1} \in[-\pi, \pi]$.

The ground state is obtained by arranging the $I_{i}^{1,2}$, s densely around zero, i.e., by choosing $M^{1}=2 M$ and $M^{2}=M$ for a chain of length $N=3 M$. Excitations are created by introducing holes into these sets of quantum numbers [41], e.g. for $N=3 M-1$ we obtain a single hole $I_{\mathrm{h}}^{2}$ in the rank-2 quantum numbers by choosing $M^{1}=2 M-1$ and $M^{2}=M-1$. In all practical applications we have to take the thermodynamic limit, such that the ABA equations (63) turn into integral equations. We introduce pseudomomenta densities

$$
\sigma^{1}\left(k_{i}^{1}\right)=\frac{1}{k_{i+1}^{1}-k_{i}^{1}}, \quad \sigma^{2}\left(k_{i}^{2}\right)=\frac{1}{k_{i+1}^{2}-k_{i}^{2}},
$$

as well as bare hole densities $\sigma_{\mathrm{h}}^{1,2}=\sum_{j} \delta\left(k-\lambda_{j}^{1,2}\right)$ which describe the excitations. Then the ABA equations (63) turn into integral equations with $\delta$-functions as integral kernels

$$
\begin{aligned}
\sigma^{1}(k)+\sigma_{\mathrm{h}}^{1}(k) & =\frac{N}{2 \pi}-\sigma^{1}(k)+\sigma^{2}(k), \\
\sigma^{2}(k)+\sigma_{\mathrm{h}}^{2}(k) & =\sigma^{1}(k)-\sigma^{2}(k) .
\end{aligned}
$$

The latter are a special and distinguishing feature of the HSM. For the ground state we have $\sigma_{\mathrm{h}}^{1,2}=0$ and (68) is solved by $\sigma_{0}^{1}=N / 3 \pi$ and $\sigma_{0}^{2}=N / 6 \pi$ [39]. This shows the simple nature of the ground state, which is explicitly given by the Gutzwiller state (6). It is, however, not possible to derive the Gutzwiller wave function from the ground state densities $\sigma_{0}^{1,2}$ as there is no direct connection between solutions of the ABA equations and the exact eigenstates of the Hamiltonian. 
One-coloron states correspond to single holes in the rank-2 pseudomomenta, i.e., $\sigma_{\mathrm{h}}^{1}=0$ and $\sigma_{\mathrm{h}}^{2}=\delta\left(k-\lambda^{2}\right)$, which implies

$$
\sigma^{1}(k)=\sigma_{0}^{1}-\frac{1}{3} \sigma_{\mathrm{h}}^{2}, \quad \sigma^{2}(k)=\sigma_{0}^{2}-\frac{2}{3} \sigma_{\mathrm{h}}^{2}
$$

There is no "hole-dressing" in the surrounding pseudomomenta, or equivalently, the pseudomomenta densities are only affected through trivial shifts as we introduce holes. This observation reflects once again the fact that colorons in the SU(3) HSM (as well as spinons in the HSM) are free [38]. The densities (69) yield the correct coloron momenta (29) and energies (30) in the thermodynamic limit. Holes in the rank-2 pseudomomenta represent excitations which transform under $\overline{3}$ [39], hence we find again that colorons possess complementary colors [14]. We wish to stress, however, that this result cannot be derived using the ABA exclusively. First, it cannot be answered whether rank-1 or rank-2 holes represent elementary excitation, and second, the ABA is not an exact method in contrast to calculations based on explicit wave functions. From (69) we can further read off that when adding one coloron, the number of available orbitals described by $\sigma^{2}(k)$ is reduced by $2 / 3$. It is, however, not completely clear to us whether this can be interpreted directly as a derivation of the exclusion statistics of polarized colorons.

\section{GENERALIZATION TO SU $(n)$}

The results derived here generalize directly to the $\mathrm{SU}(n)$ HSM. Consider a chain with one particle per lattice site carrying an internal $\mathrm{SU}(n)$ quantum number which transforms according to the fundamental representation $n$ of $\mathrm{SU}(n)$. The Hamiltonian is given by

$$
H_{\mathrm{SU}(n)}=\frac{2 \pi^{2}}{N^{2}} \sum_{\alpha<\beta}^{N} \sum_{\sigma \tau}^{n} \frac{e_{\alpha}^{\sigma \tau} e_{\beta}^{\tau \sigma}}{\left|\eta_{\alpha}-\eta_{\beta}\right|^{2}},
$$

where the operator $e_{\alpha}^{\sigma \tau}$ annihilates a particle of flavor $\tau$ at site $\eta_{\alpha}$ and creates a particle of flavor $\sigma$ at the same site. (Once again, $\tau$ and $\sigma$ may be equal in (70). In comparison to (2) , we have omitted a constant.) The model again possesses a Yangian symmetry [18] and hence is integrable.

If we use a polarized state of particles of flavor $n$ as reference state and label the coordinates of the particles of flavor $\sigma, 1 \leq \sigma \leq n-1$, by $z_{i}^{\sigma}, 1 \leq i \leq M_{\sigma}$, the wave functions [20] 


$$
\Psi_{0}\left[z_{i}^{\sigma}\right]=\prod_{\sigma=1}^{n-1} \prod_{i<j}^{M_{\sigma}}\left(z_{i}^{\sigma}-z_{j}^{\sigma}\right)^{2} \prod_{\sigma<\tau}^{n-1} \prod_{i=1}^{M_{\sigma}} \prod_{j=1}^{M_{\tau}}\left(z_{i}^{\sigma}-z_{j}^{\tau}\right) \prod_{\sigma=1}^{n-1} \prod_{i=1}^{M_{\sigma}} z_{i}^{\sigma}
$$

constitute exact eigenstates [21] of the Hamiltonian (170). For $N=n M, M_{\sigma}=M$, (171) is the ground state of (170) with energy [20]

$$
E_{0}^{n}=-\frac{\pi^{2}}{12}\left(\frac{n-2}{n} N+\frac{2 n-1}{N}\right) .
$$

The total momentum of this ground state is $p=(n-1) \pi M \bmod 2 \pi$.

Localized $\mathrm{SU}(n)$ spinons are given by

$$
\Psi_{\gamma}\left[z_{i}^{\sigma}\right]=\prod_{i=1}^{M_{1}}\left(\eta_{\gamma}-z_{i}^{1}\right) \Psi_{0}\left[z_{i}^{\sigma}\right]
$$

for $N=n M-1, M_{1}=M-1$, and $M_{2}=\ldots=M_{n-1}=M$. As hole-like excitations they transform according to the representation $\bar{n}$. Energy eigenstates are obtained by Fourier transformation of (173),

$$
\Psi_{m}\left[z_{i}^{\sigma}\right]=\frac{1}{N} \sum_{\gamma=1}^{N}\left(\bar{\eta}_{\gamma}\right)^{m} \Psi_{\gamma}\left[z_{i}^{\sigma}\right], \quad 0 \leq m \leq M_{1} .
$$

Their momenta are given by

$$
p=\frac{n-1}{n} \pi N-\frac{2 \pi}{N}\left(m+\frac{n-1}{2 n}\right) \bmod 2 \pi,
$$

which fill the interval $\left[-\frac{\pi}{n}, \frac{\pi}{n}\right]$ for $n$ even and $M$ odd, or the interval $\left[-\frac{\pi}{n}, \frac{\pi}{n}\right]$ otherwise (either $n$ odd or $M$ even or both). The energy eigenvalues of the one $\mathrm{SU}(n)$ spinon states (74) are given by

$$
E^{n}(p)=E_{0}^{n}+\frac{n^{2}-1}{12 n} \frac{\pi^{2}}{N^{2}}+\epsilon^{n}(p)
$$

with (see Fig. 5)

$$
\epsilon^{n}(p)= \begin{cases}\frac{n}{4}\left(\frac{\pi^{2}}{n^{2}}-p^{2}\right), & \text { if } n \text { even and } M \text { odd } \\ \frac{n}{4}\left(\frac{\pi^{2}}{n^{2}}-(p-\pi)^{2}\right), & \text { otherwise. }\end{cases}
$$

The statistical parameter for polarized $\mathrm{SU}(n)$ spinons is $g=(n-1) / n$. In the framework of the ABA, the spinons are represented by holes in rank- $(n-1)$ pseudomomenta. 
a) $n$ even

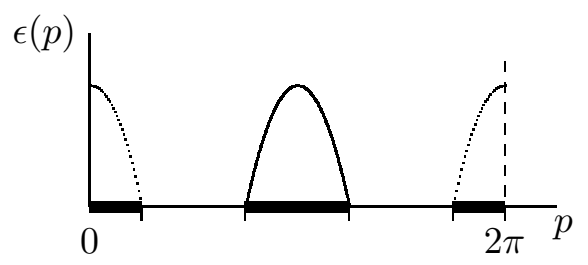

b) $n$ odd

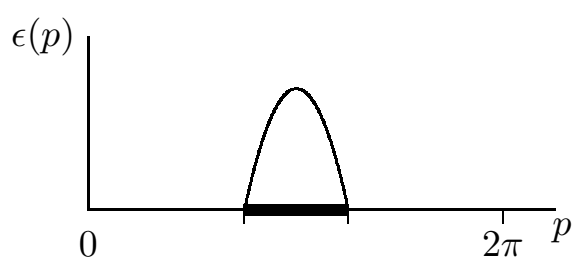

FIG. 5: $\mathrm{SU}(n)$ spinon dispersion relations. a) $n$ even. The allowed momenta fill the interval $\left[\pi-\frac{\pi}{n}, \pi+\frac{\pi}{n}\right]$ for $M$ even and $\left[-\frac{\pi}{n}, \frac{\pi}{n}\right]$ for $M$ odd. b) $n$ odd. The allowed momenta fill the interval $\left[\pi-\frac{\pi}{n}, \pi+\frac{\pi}{n}\right]$

Finally, let us add some remarks on the large- $n$ limit of the $\mathrm{SU}(n)$ HSM. First, there are no simplifications in the calculations which yield the results presented in this section. When $n$ grows, we obtain solely terms similar to the ones treated in the appendix. Second, the interval of allowed spinon momenta shrinks to zero. This does not mean that the number of spinon orbitals vanishes, as in the limit $n \rightarrow \infty$ the number of lattice sites $N=n M-1$ has to grow and hence the momentum spacing tends to zero as well. Third, we find $g \rightarrow 1$ as $n \rightarrow \infty$, meaning that the exclusion statistics between polarized spinons becomes fermionic in this limit. This does not imply, however, that spinons in the $\mathrm{SU}(n) \mathrm{HSM}$ behave like free fermions in the large- $n$ limit. Their momenta fill only a narrow interval and the individual spinon momenta are not simply given by integer multiples of $2 \pi / N$ [34].

\section{CONCLUSION}

In conclusion, we have shown by explicit calculation that the elementary excitations of $\mathrm{SU}(n)$, but in particular $\mathrm{SU}(3)$, HSM transform under the representation conjugate to the representation of the $\mathrm{SU}(n)$ spins on the chain. We have also shown that they obey fractional statistics, and only exist in one $\mathrm{n}^{\text {th }}$ of the Brillouin zone. We strongly believe these features to be generic features of "antiferromagnetic" $\mathrm{SU}(n)$ spin chains. We have further endeavored to construct the polarized two-coloron eigenstates of the HSM explicitly, and have thereby shown that they are free and that the spacings in the difference of the individual coloron momenta $p_{n}$ and $p_{m}$ with $p_{n} \geq p_{m}$ are given by $p_{n}-p_{m}=\frac{2 \pi}{N}\left(\frac{2}{3}+\right.$ integer $)$. We have interpreted this peculiar shift as a manifestation of the fractional statistics. This indicates that fractional statistics in one dimension manifests itself not only through a fractional 
exclusion principle [24], but that there are Berry's phases associated with anyons [23] in one dimensions as well [40]. Furthermore, we look forward to an experimental realization of $\mathrm{SU}(3)$ spin chains in systems of ultracold atoms [12, 13], and hence an observation of the complementary colors of the excitations.

\section{ACKNOWLEDGMENTS}

MG would like to thank the organizers of the 2003 Amsterdam Summer Workshop on Flux, Charge, Topology and Statistics, where this work was partially inspired. DS was supported by the German Research Foundation (DFG) through GK 284.

\section{APPENDIX A: GELL-MANN MATRICES}

The Gell-Mann matrices are given by [25]

$$
\begin{aligned}
& \lambda^{1}=\left(\begin{array}{lll}
0 & 1 & 0 \\
1 & 0 & 0 \\
0 & 0 & 0
\end{array}\right), \quad \lambda^{2}=\left(\begin{array}{ccc}
0 & -i & 0 \\
i & 0 & 0 \\
0 & 0 & 0
\end{array}\right), \quad \lambda^{3}=\left(\begin{array}{ccc}
1 & 0 & 0 \\
0 & -1 & 0 \\
0 & 0 & 0
\end{array}\right), \\
& \lambda^{4}=\left(\begin{array}{lll}
0 & 0 & 1 \\
0 & 0 & 0 \\
1 & 0 & 0
\end{array}\right), \quad \lambda^{5}=\left(\begin{array}{ccc}
0 & 0 & -i \\
0 & 0 & 0 \\
i & 0 & 0
\end{array}\right), \quad \lambda^{6}=\left(\begin{array}{lll}
0 & 0 & 0 \\
0 & 0 & 1 \\
0 & 1 & 0
\end{array}\right), \\
& \lambda^{7}=\left(\begin{array}{lll}
0 & 0 & 0 \\
0 & 0 & -i \\
0 & i & 0
\end{array}\right), \quad \lambda^{8}=\frac{1}{\sqrt{3}}\left(\begin{array}{ccc}
1 & 0 & 0 \\
0 & 1 & 0 \\
0 & 0 & -2
\end{array}\right) .
\end{aligned}
$$

They are normalized as $\operatorname{tr}\left(\lambda^{a} \lambda^{b}\right)=2 \delta_{a b}$ and satisfy the commutation relations $\left[\lambda^{a}, \lambda^{b}\right]=$ $2 f^{a b c} \lambda^{c}$. The structure constants $f^{a b c}$ are totally antisymmetric and obey Jacobi's identity

$$
f^{a b c} f^{c d e}+f^{b d c} f^{c a e}+f^{d a c} f^{c b e}=0
$$

Explicitly, the non-vanishing structure constants are given by $f^{123}=i, f^{147}=f^{246}=f^{257}=$ $f^{345}=-f^{156}=-f^{367}=i / 2, f^{458}=f^{678}=i \sqrt{3} / 2$, and 45 others obtained by permutations 
of the indices. The $\mathrm{SU}(3)$ spin operators are expressed in terms of the colorflip operators by

$$
\boldsymbol{J}_{\alpha} \cdot \boldsymbol{J}_{\beta} \equiv \sum_{a=1}^{8} J_{\alpha}^{a} J_{\beta}^{a}=\frac{1}{2} \sum_{\sigma \tau}^{3} e_{\alpha}^{\sigma \tau} e_{\beta}^{\tau \sigma}-\frac{1}{6}
$$

\section{APPENDIX B: EQUIVALENCE OF (6) AND (9)}

Let us begin by exploring a general consequence of particle-hole symmetry. Consider a chain of $N$ sites. The wave function of $L$ fermions with momenta in the interval $I_{1}$ is given by

$$
\Phi_{1}\left[u_{1}, \ldots, u_{L}\right]=\left\langle 0\left|c_{u_{1}} \cdots c_{u_{L}} \prod_{q \in I_{1}} c_{q}^{\dagger}\right| 0\right\rangle .
$$

This wave function can be expressed in terms of $N-L$ fermions whose momenta fill the interval $I_{2}=[-\pi, \pi] \backslash I_{1}$,

$$
\begin{aligned}
\Phi_{1}\left[u_{1}, \ldots, u_{L}\right] & =\left(\left\langle 0\left|\prod_{q \in I_{1}} c_{q} c_{u_{L}}^{\dagger} \cdots c_{u_{1}}^{\dagger}\right| 0\right\rangle\right)^{*} \\
& =\operatorname{sign} \cdot\left(\left\langle 0\left|\prod_{q \in I_{1}} c_{q} c_{v_{1}} \cdots c_{v_{N-L}} \prod_{q \in I_{2}} c_{q}^{\dagger} \prod_{q \in I_{1}} c_{q}^{\dagger}\right| 0\right\rangle\right)^{*} \\
& =\operatorname{sign} \cdot \Phi_{2}^{*}\left[v_{1}, \ldots, v_{N-L}\right],
\end{aligned}
$$

where the coordinates $v_{1}, \ldots, v_{N-L}$ are those coordinates on the chain which are not contained in the set $u_{1}, \ldots, u_{L}$,

$$
\Phi_{2}\left[v_{1}, \ldots, v_{N-L}\right]=\left\langle 0\left|c_{v_{1}} \cdots c_{v_{N-L}} \prod_{q \in I_{2}} c_{q}^{\dagger}\right| 0\right\rangle
$$

and the overall sign depends on the number and positions of the fermions.

(B2) enables us to show that the ground state wave function is given by (91) with $M_{1}=$ $M_{2}=M$. For $N=3 M$, the wave function of the Slater determinant state $\left|\Psi_{\mathrm{SD}}\right\rangle$ is (see Fig. 2a)

$$
\Psi_{\mathrm{SD}}\left[z_{i} ; w_{k} ; u_{m}\right]=\Phi_{1}\left[z_{i}\right] \Phi_{1}\left[w_{k}\right] \Phi_{1}\left[u_{m}\right]
$$

where the interval $I_{1}$ is chosen to be $[-\pi / 3, \pi / 3]$ and hence

$$
\Phi_{1}\left[z_{i}\right]=\prod_{i=1}^{M} z_{i}^{-\frac{M-1}{2}} \prod_{i<j}^{M}\left(z_{i}-z_{j}\right) .
$$

The prefactor in (B5) shifts all momenta from the interval $[0,2 \pi / 3]$ to $I_{1}$. The ground state

of the $\mathrm{SU}(3) \mathrm{HSM}$ is obtained by Gutzwiller projection, $\left|\Psi_{0}\right\rangle=P_{\mathrm{G}}\left|\Psi_{\mathrm{SD}}\right\rangle$, which enforces 
that all coordinates $z_{i}, w_{k}$, and $u_{m}$ are distinct. Hence, we can express the coordinates $u_{m}$ in terms of the $z_{i}$ 's and $w_{k}$ 's using (Bי

$$
\Phi_{1}\left[u_{m}\right]=\operatorname{sign} \cdot \Phi_{2}^{*}\left[z_{i}, w_{k}\right]
$$

where the wave function of the filled band with momenta in the interval $I_{2}=[-\pi, \pi] \backslash I_{1}$ is (up to a sign) given by

$$
\Phi_{2}\left[z_{i}, w_{k}\right]=\prod_{i=1}^{M} z_{i}^{\frac{M+1}{2}} \prod_{k=1}^{M} w_{k}^{\frac{M+1}{2}} \prod_{i<j}^{M}\left(z_{i}-z_{j}\right) \prod_{k<l}^{M}\left(w_{k}-w_{l}\right) \prod_{i=1}^{M} \prod_{k=1}^{M}\left(z_{i}-w_{k}\right) .
$$

The ground state wave function is hence given by

$$
\begin{aligned}
\Psi_{0}\left[z_{i}, w_{k}\right] & =\Phi_{1}\left[z_{i}\right] \Phi_{1}\left[w_{k}\right] \Phi_{2}^{*}\left[z_{i}, w_{k}\right] \\
& =\prod_{i=1}^{M} z_{i}^{-M} \prod_{k=1}^{M} w_{k}^{-M} \prod_{i<j}^{M}\left|z_{i}-z_{j}\right|^{2} \prod_{k<l}^{M}\left|w_{k}-w_{l}\right|^{2} \prod_{i=1}^{M} \prod_{k=1}^{M}\left(z_{i}-w_{k}\right)^{*}
\end{aligned}
$$

where we have used the fact that the coordinates are located on the unit circle, e.g. $z_{i}^{*}=1 / z_{i}$. Using

$$
\left|z_{i}-z_{j}\right|^{2}=-\frac{\left(z_{i}-z_{j}\right)^{2}}{z_{i} z_{j}}, \quad \prod_{i<j}^{M} \frac{1}{z_{i} z_{j}}=\prod_{i=1}^{M} \frac{1}{z_{i}^{M-1}}
$$

as well as

$$
\left(z_{i}-w_{k}\right)^{*}=-\frac{\left(z_{i}-w_{k}\right)}{z_{i} w_{k}}, \quad \prod_{i=1}^{M} \prod_{k=1}^{M} \frac{1}{z_{i} w_{k}}=\prod_{i=1}^{M} \frac{1}{z_{i}^{M}} \prod_{k=1}^{M} \frac{1}{w_{k}^{M}}
$$

we obtain from (B7)

$$
\Psi_{0}\left[z_{i}, w_{k}\right]=\prod_{i=1}^{M} z_{i}^{-3 M} \prod_{k=1}^{M} w_{k}^{-3 M} \prod_{i<j}^{M}\left(z_{i}-z_{j}\right)^{2} \prod_{k<l}^{M}\left(w_{k}-w_{l}\right)^{2} \prod_{i=1}^{M} \prod_{k=1}^{M}\left(z_{i}-w_{k}\right) \prod_{i=1}^{M} z_{i} \prod_{k=1}^{M} w_{k} .
$$

As the coordinates $z_{i}$ and $w_{k}$ are located at the lattice sites $\eta_{\alpha}=\exp \left(\frac{2 \pi i}{N} \alpha\right)$ and $N=3 M$, the prefactor reduces to one and $\Psi_{0}\left[z_{i}, w_{k}\right]$ takes the form (91).

\section{APPENDIX C: USEFUL FORMULAS}

Some of the results presented in this appendix can be found in [35].

1.

$$
\eta_{\alpha}^{N}=1, \quad \sum_{\alpha=1}^{N} \eta_{\alpha}^{m}=N \delta_{0 m}, \quad \prod_{\alpha=1}^{N} \eta_{\alpha}=(-1)^{N-1}
$$



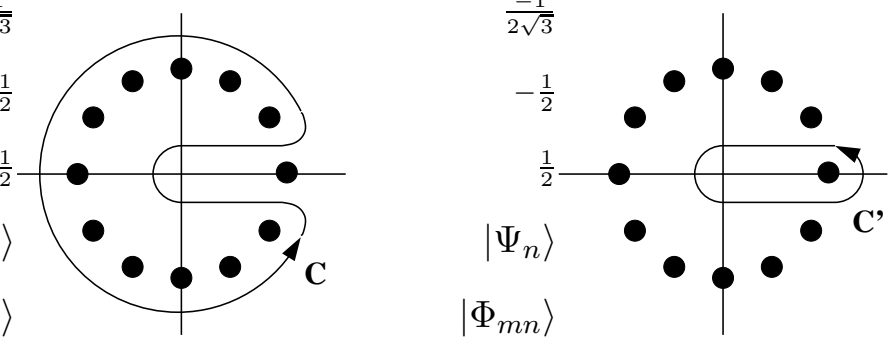

FIG. 6: Contours for integrations

2.

$$
\prod_{\alpha=1}^{N}\left(z-\eta_{\alpha}\right)=z^{N}-1
$$

3.

$$
\sum_{\alpha=1}^{N} \frac{\eta_{\alpha}}{z-\eta_{\alpha}}=\frac{N}{z^{N}-1}
$$

Proof: Rewrite (C3) as

$$
f(z) \equiv \sum_{\alpha=1}^{N} \eta_{\alpha} \prod_{\beta \neq \alpha}^{N}\left(z-\eta_{\beta}\right)=N
$$

with $f$ a polynomial of degree $N-1, f(0)=N$, and $f\left(1 / \eta_{\gamma}\right)=\prod_{\beta=1}^{N-1}\left(1-\eta_{\beta}\right), \gamma=$ $1, \ldots, N$. Since the polynomial $f-f(1)$ has degree $N-1$ and $N$ different zeros, it vanishes by the fundamental theorem of algebra [42].

4. The previous statement implies

$$
\sum_{\alpha=1}^{N} \frac{1}{\eta-\eta_{\alpha}}=\frac{N \eta^{N-1}}{\eta^{N}-1}
$$

5.

$$
\sum_{\alpha=1}^{N-1} \frac{\eta_{\alpha}^{m}}{\eta_{\alpha}-1}=\frac{N+1}{2}-m, \quad 1 \leq m \leq N .
$$

Proof: Using Cauchy's theorem [42] for the function

$$
f(z)=\frac{z^{m}}{z^{N}(z-1)}, \quad 2 \leq N
$$

with the contours drawn in Fig. 6 yields

$$
\sum_{\alpha=1}^{N-1} \frac{\eta_{\alpha}^{m}}{\eta_{\alpha}-1}=\frac{1}{2 \pi i} \sum_{\alpha=1}^{N-1} \oint_{C} \frac{z^{m}}{z^{N}(z-1)} \frac{1}{z-\eta_{\alpha}} d z
$$




$$
\begin{aligned}
& =\frac{N}{2 \pi i} \oint_{C} \frac{z^{m-1}}{(z-1)\left(z^{N}-1\right)} d z \\
& =-\frac{N}{2 \pi i} \oint_{C^{\prime}} \frac{z^{m-1}}{(z-1)\left(z^{N}-1\right)} d z \\
& =\frac{N+1}{2}-m,
\end{aligned}
$$

where we have used the theorem of residues [42] in the last step.

6.

$$
\sum_{\alpha=1}^{N-1} \frac{\eta_{\alpha}^{m}}{\left|\eta_{\alpha}-1\right|^{2}}=\frac{N^{2}-1}{12}-\frac{m(N-1)}{2}+\frac{m(m-1)}{2}, \quad 0 \leq m \leq N .
$$

Proof: We have

$$
\begin{aligned}
\sum_{\alpha=1}^{N-1} \frac{\eta_{\alpha}^{m}}{\left|\eta_{\alpha}-1\right|^{2}} & =-\sum_{\alpha=1}^{N-1} \frac{\eta_{\alpha}^{m+1}}{\left(\eta_{\alpha}-1\right)^{2}} \\
& =-\frac{N}{2 \pi i} \oint_{C} \frac{z^{m}}{(z-1)^{2}\left(z^{N}-1\right)} d z \\
& =\frac{N}{2 \pi i} \oint_{C^{\prime}} \frac{z^{m}}{(z-1)^{2}\left(z^{N}-1\right)} d z \\
& =\frac{N^{2}-1}{12}-\frac{m(N-1)}{2}+\frac{m(m-1)}{2} .
\end{aligned}
$$

7. For

$$
A_{m}=-\sum_{\alpha=1}^{N-1} \eta_{\alpha}^{2}\left(\eta_{\alpha}-1\right)^{m-2}
$$

we have: $A_{0}=(N-1)(N-5) / 12$ by (C6) $), A_{1}=-(N-3) / 2$ by (C5), and $A_{2}=1$ by $\sum_{\alpha}^{N} \eta_{\alpha}^{m}=N \delta_{m 0}$. Furthermore,

$$
\begin{aligned}
A_{m} & =-\sum_{\alpha=1}^{N-1} \eta_{\alpha}^{2} \sum_{k=0}^{m-2}\left(\begin{array}{c}
m-2 \\
k
\end{array}\right)(-1)^{m-k-2} \eta_{\alpha}^{k} \\
& =\sum_{k=0}^{m-2}\left(\begin{array}{c}
m-2 \\
k
\end{array}\right)(-1)^{m-k}\left(1-\sum_{\alpha=1}^{N} \eta_{\alpha}^{k+2}\right) \\
& =\sum_{k=0}^{n}\left(\begin{array}{l}
n \\
k
\end{array}\right)(-1)^{n-k}=0, \quad 2<m \leq N-1 .
\end{aligned}
$$

as the sums of the binomial coefficients of even sites and odd sites equal each other.

8. For

$$
B_{m}=\sum_{\alpha=1}^{N-1} \eta_{\alpha}\left(\eta_{\alpha}+1\right)\left(\eta_{\alpha}-1\right)^{m-1}
$$

we have: $B_{0}=N-2, B_{1}=-2, B_{m}=0$ for $2<m \leq N-2$, and $B_{N-1}=N$. 


\section{APPENDIX D: PROOF OF THEOREM 1}

As (22) is symmetric in the $z_{i}^{\prime}$ 's, we prove the theorem by showing that the LHS of (22) is independent of $z_{1}$ and vanishes identically. To this end, consider the auxiliary function

$$
\Phi\left(z_{1}\right)=\frac{z_{1}\left(z_{1}-z\right)^{M-3}}{\prod_{j=2}^{M}\left(z_{j}-z_{1}\right)}+\sum_{i=2}^{M} \frac{z_{i}\left(z_{i}-z\right)^{M-3}}{\left(z_{1}-z_{i}\right) \prod_{j \neq i}^{M}\left(z_{j}-z_{i}\right)} .
$$

for fixed and distinct $z_{2}, \ldots, z_{M} \in \mathbb{C}$.

First, we show that (D1) is entire (i.e., analytic on $\mathbb{C}$ ). We can restrict ourselves to showing that $\Phi\left(z_{1}\right)$ is analytic at $z_{1}=z_{2}$. (D1) can be rewritten as

$$
\Phi\left(z_{1}\right)=-\frac{z_{1}\left(z_{1}-z\right)^{M-3} \prod_{j=3}^{M}\left(z_{j}-z_{2}\right)-z_{2}\left(z_{2}-z\right)^{M-3} \prod_{j=3}^{M}\left(z_{j}-z_{1}\right)}{\left(z_{1}-z_{2}\right) \prod_{j=3}^{M}\left(z_{j}-z_{1}\right)\left(z_{j}-z_{2}\right)}+\tilde{\Phi}\left(z_{1}\right)
$$

where $\tilde{\Phi}$ is analytic at $z_{1}=z_{2}$. Expanding the first term yields expressions like

$$
\frac{1}{z_{1}-z_{2}}\left(z_{1}^{n} z_{2}^{m}-z_{1}^{m} z_{2}^{n}\right), \quad m, n \in \mathbb{N}
$$

which can be shown to be analytic at $z_{1}=z_{2}$ by using (39).

Second, as the degree of the denominator in (D1) is strictly greater than the degree of the numerator, $\Phi\left(z_{1}\right)$ vanishes for $\left|z_{1}\right| \rightarrow \infty$. Therefore, by Liouville's theorem [42] $\Phi$ vanishes for all $z_{1} \in \mathbb{C}$, which proves the theorem.

\section{APPENDIX E: PROOF OF THEOREM 3}

We prove the theorem in three steps. First, we show that the LHS of (36) does not depend on the $w_{k}$ 's. Second, we eliminate the $w_{k}$ 's. Third, we verify the remaining equation by explicit calculation.

For the first step, note that (36) is symmetric under permutations of the $w_{k}$ 's. It is hence sufficient to show that (36) does not depend on $w_{1}$. Consider for fixed and distinct $z_{1}, \ldots, z_{M_{1}}, w_{2}, \ldots, w_{M_{2}} \in \mathbb{C}$ the auxiliary function

$$
\begin{aligned}
\Phi\left(w_{1}\right)= & \frac{1}{z_{1}-w_{1}}\left[w_{1} \prod_{j=2}^{M_{1}} \frac{z_{j}-w_{1}}{z_{j}-z_{1}} \prod_{l=2}^{M_{2}} \frac{w_{l}-z_{1}}{w_{l}-w_{1}}-z_{1}\right] \\
& +\sum_{k=2}^{M_{2}} \frac{1}{z_{1}-w_{k}}\left[w_{k} \prod_{j=2}^{M_{1}} \frac{z_{j}-w_{k}}{z_{j}-z_{1}} \prod_{l \neq k}^{M_{2}} \frac{w_{l}-z_{1}}{w_{l}-w_{k}} \cdot \frac{w_{1}-z_{1}}{w_{1}-w_{k}}-z_{1}\right] .
\end{aligned}
$$


We will show that $\Phi$ is an entire function and bounded for $\left|w_{1}\right| \rightarrow \infty$. Then Liouville's theorem [42] implies that $\Phi$ is a constant, i.e., independent of $w_{1}$.

To see that $\Phi$ is entire note that (E1) is obviously analytic for $w_{1} \in \mathbb{C} \backslash\left\{z_{1}, w_{2}, \ldots, w_{M_{2}}\right\}$. To investigate the point $w_{1}=z_{1}$, we rewrite the first line of (E1) as

$$
\frac{1}{z_{1}-w_{1}}\left[w_{1} \prod_{j=2}^{M_{1}}\left(1+\frac{z_{1}-w_{1}}{z_{j}-z_{1}}\right) \prod_{l=2}^{M_{2}}\left(1-\frac{z_{1}-w_{1}}{w_{l}-w_{1}}\right)-z_{1}\right]
$$

which shows that $\Phi$ can be analytically continued to $w_{1}=z_{1}$. Furthermore, as (E1) is symmetric in $w_{2}, \ldots, w_{M_{2}}$, we may restrict ourselves now to showing that $\Phi$ is analytic at $w_{1}=w_{2}$. For $w_{2}=0$ this is obviously the case. For $w_{2} \neq 0$ we write $\Phi$ as

$$
\begin{aligned}
\Phi\left(w_{1}\right)= & \frac{w_{1}}{z_{1}-w_{1}} \prod_{j=2}^{M_{1}} \frac{z_{j}-w_{1}}{z_{j}-z_{1}} \prod_{l=3}^{M_{2}} \frac{w_{l}-z_{1}}{w_{l}-w_{1}} \cdot \frac{w_{2}-z_{1}}{w_{2}-w_{1}} \\
& +\frac{w_{2}}{z_{1}-w_{2}} \prod_{j=2}^{M_{1}} \frac{z_{j}-w_{2}}{z_{j}-z_{1}} \prod_{l=3}^{M_{2}} \frac{w_{l}-z_{1}}{w_{l}-w_{2}} \cdot \frac{w_{1}-z_{1}}{w_{1}-w_{2}}+\tilde{\Phi}\left(w_{1}\right),
\end{aligned}
$$

where $\tilde{\Phi}$ is analytic at $w_{1}=w_{2}$. Now, $\Phi-\tilde{\Phi}$ can be rewritten as

$$
\begin{aligned}
& \frac{1}{\left(z_{1}-w_{1}\right)\left(z_{1}-w_{2}\right)} \prod_{j=2}^{M_{1}} \frac{1}{z_{j}-z_{1}} \prod_{l=3}^{M_{2}} \frac{w_{l}-z_{1}}{\left(w_{l}-w_{1}\right)\left(w_{l}-w_{2}\right)} \frac{1}{w_{1}-w_{2}} \\
& \cdot\left[w_{1}\left(z_{1}-w_{2}\right)^{2} \prod_{j=2}^{M_{1}}\left(z_{j}-w_{1}\right) \prod_{l=3}^{M_{2}}\left(w_{l}-w_{2}\right)-w_{2}\left(z_{1}-w_{1}\right)^{2} \prod_{j=2}^{M_{1}}\left(z_{j}-w_{2}\right) \prod_{l=3}^{M_{2}}\left(w_{l}-w_{1}\right)\right] .
\end{aligned}
$$

Expanding the square brackets in (E2) leads to terms like

$$
\frac{1}{w_{1}-w_{2}}\left(w_{1}^{n} w_{2}^{m}-w_{1}^{m} w_{2}^{n}\right), \quad m, n \in \mathbb{N}
$$

which can be shown to be analytic at $w_{1}=w_{2}$ by using (39). This concludes the proof that $\Phi$ is an entire function of $w_{1}$. Furthermore, $\Phi$ tends to a constant for $\left|w_{1}\right| \rightarrow \infty$ as in the first term of (E1), the degree of the denominator is greater or equal than the degree of the numerator, while in the second term the $w_{1}$-dependence cancels out for $\left|w_{1}\right| \rightarrow \infty$. Therefore, $\Phi$ is constant by Liouville's theorem [42]. As we can replace $z_{1}$ by any $z_{i}$ in (E1), the LHS of (36) does not depend on the $w_{k}$ 's.

In the second step, we eliminate the $w_{k}$ 's from (36). In order to do so we choose $w_{j}=z_{j}$ for $1 \leq j \leq M_{1}$, and $w_{k}=R e^{i \varphi_{k}}$ with distinct values $\varphi_{k} \in[0,2 \pi]$ for $M_{1}+1 \leq k \leq M_{2}$. We 
now have to study three different terms in (36). First, the terms with $i=k$ yield as $R \rightarrow \infty$

$$
-\sum_{i=1}^{M_{1}} z_{i} \sum_{\gamma=1}^{N}\left(\bar{\eta}_{\gamma}\right)^{n} \prod_{j \neq i}^{M_{1}}\left(\eta_{\gamma}-z_{j}\right)
$$

Second, the terms with $i \neq k, k \leq M_{1}$ yield in the same limit

$$
-\sum_{i=1}^{M_{1}} \sum_{k \neq i}^{M_{1}} \frac{z_{i}^{2}}{z_{i}-z_{k}} \sum_{\gamma=1}^{N}\left(\bar{\eta}_{\gamma}\right)^{n} \prod_{j \neq i}^{M_{1}}\left(\eta_{\gamma}-z_{j}\right) .
$$

Third, the terms with $M_{1}<k$ vanish in this limit. Hence (36) reduces to

$$
\begin{gathered}
\sum_{i=1}^{M_{1}} z_{i} \sum_{\gamma=1}^{N}\left(\bar{\eta}_{\gamma}\right)^{n} \prod_{k \neq i}^{M_{1}}\left(\eta_{\gamma}-z_{k}\right)+\sum_{i=1}^{M_{1}} \sum_{j \neq i}^{M_{1}} \frac{z_{i}^{2}}{z_{i}-z_{j}} \sum_{\gamma=1}^{N}\left(\bar{\eta}_{\gamma}\right)^{n} \prod_{k \neq i}^{M_{1}}\left(\eta_{\gamma}-z_{k}\right) \\
=-f(n) \sum_{\gamma=1}^{N}\left(\bar{\eta}_{\gamma}\right)^{n} \prod_{i=1}^{M_{1}}\left(\eta_{\gamma}-z_{i}\right),
\end{gathered}
$$

with $f(n)=-n(n+1) / 2+M_{1}\left(M_{1}+1\right) / 2$. The second term in the LHS of (E3) can be written as

$$
\begin{aligned}
\sum_{\gamma=1}^{N}\left(\bar{\eta}_{\gamma}\right)^{n} & \sum_{i \neq j}^{M_{1}} \frac{z_{i}^{2}}{z_{i}-z_{j}}\left(\eta_{\gamma}-z_{j}\right) \prod_{k \neq i, j}^{M_{1}}\left(\eta_{\gamma}-z_{k}\right) \\
& =\sum_{\gamma=1}^{N}\left[\left(\bar{\eta}_{\gamma}\right)^{n-1} \sum_{i \neq j}^{M_{1}} z_{i}-\frac{1}{2}\left(\bar{\eta}_{\gamma}\right)^{n} \sum_{i \neq j}^{M_{1}} z_{i} z_{j}\right] \prod_{k \neq i, j}^{M_{1}}\left(\eta_{\gamma}-z_{k}\right),
\end{aligned}
$$

where we have used

$$
\sum_{i \neq j}^{M_{1}} \frac{z_{i}^{2}}{z_{i}-z_{j}}\left(\eta_{\gamma}-z_{j}\right)=\frac{1}{2} \sum_{i \neq j}^{M_{1}}\left[\eta_{\gamma}\left(z_{i}+z_{j}\right)-z_{i} z_{j}\right] .
$$

In the third step, we complete the proof by explicit calculation. For this we use

$$
\sum_{\gamma=1}^{N}\left(\bar{\eta}_{\gamma}\right)^{n} \prod_{i=1}^{M_{1}}\left(\eta_{\gamma}-z_{i}\right)=(-1)^{M_{1}-n} \mathcal{S}^{M_{1}}\left(z_{1} \cdots z_{M_{1}-n}\right)
$$

where $\mathcal{S}^{M_{1}}\left(z_{1} \cdots z_{M_{1}-n}\right)$ is the sum over all possible ways to choose $M_{1}-n$ coordinates out of $z_{1}, \ldots, z_{M_{1}}$. Then, using (E4) we write (E3) as

$$
\begin{aligned}
& \left.\sum_{i=1}^{M_{1}} z_{i} \mathcal{S}^{M_{1}-1}\left(z_{1} \cdots z_{M_{1}-n-1}\right)\right|_{\text {no } z_{i}}+\left.\sum_{i \neq j}^{M_{1}} z_{i} \mathcal{S}^{M_{1}-2}\left(z_{1} \cdots z_{M_{1}-n-1}\right)\right|_{\text {no } z_{i}, z_{j}} \\
& +\left.\frac{1}{2} \sum_{i \neq j}^{M_{1}} z_{i} z_{j} \mathcal{S}^{M_{1}-2}\left(z_{1} \cdots z_{M_{1}-n-2}\right)\right|_{\text {no } z_{i}, z_{j}}=f(n) \mathcal{S}^{M_{1}}\left(z_{1} \cdots z_{M_{1}-n}\right) .
\end{aligned}
$$


With the relations

$$
\begin{aligned}
\left.\sum_{i=1}^{M_{1}} z_{i} \mathcal{S}^{M_{1}-1}\left(z_{1} \cdots z_{M_{1}-n-1}\right)\right|_{\text {no } z_{i}} & =\left(M_{1}-n\right) \mathcal{S}^{M_{1}}\left(z_{1} \cdots z_{M_{1}-n}\right), \\
\left.\sum_{i \neq j}^{M_{1}} z_{i} \mathcal{S}^{M_{1}-2}\left(z_{1} \cdots z_{M_{1}-n-1}\right)\right|_{\text {no } z_{i}, z_{j}} & =\left(M_{1}-n\right) n \mathcal{S}^{M_{1}}\left(z_{1} \cdots z_{M_{1}-n}\right), \\
\left.\sum_{i \neq j}^{M_{1}} z_{i} z_{j} \mathcal{S}^{M_{1}-2}\left(z_{1} \cdots z_{M_{1}-n-2}\right)\right|_{\text {no } z_{i}, z_{j}} & =\left(M_{1}-n\right)\left(M_{1}-n-1\right) \mathcal{S}^{M_{1}}\left(z_{1} \cdots z_{M_{1}-n}\right),
\end{aligned}
$$

we can verify (E6) and deduce $f(n)=-n(n+1) / 2+M_{1}\left(M_{1}+1\right) / 2$. This concludes the proof of the theorem.

\section{APPENDIX F: PROOF OF THEOREM 4}

Consider for fixed and distinct $z_{1}, \ldots, z_{M_{1}}, w_{2}, \ldots, w_{M_{2}} \in \mathbb{C}$ the auxiliary function

$$
\begin{aligned}
\Phi\left(w_{1}\right)= & w_{1} \prod_{j=2}^{M_{1}} \frac{z_{j}-w_{1}}{z_{j}-z_{1}} \prod_{l=2}^{M_{2}} \frac{w_{l}-z_{1}}{w_{l}-w_{1}} \\
& +\sum_{k=2}^{M_{2}} w_{k} \prod_{j=2}^{M_{1}} \frac{z_{j}-w_{k}}{z_{j}-z_{1}} \prod_{l \neq k}^{M_{2}} \frac{w_{l}-z_{1}}{w_{l}-w_{k}} \cdot \frac{w_{1}-z_{1}}{w_{1}-w_{k}} .
\end{aligned}
$$

In analogy to the proof in App. E it can be shown by using (39) that (F1) is entire, and that $\Phi$ tends to a constant for $\left|w_{1}\right| \rightarrow \infty$. By Liouville's theorem [42] $\Phi$ is constant, and hence (46) is independent of the $w_{k}$ 's. We can choose the $w_{k}$ 's as $w_{j}=z_{j}$ for $1 \leq j \leq M_{1}$, and $w_{k}=R e^{i \varphi_{k}}$ with distinct values $\varphi_{k} \in[0,2 \pi]$ for $M_{1}+1 \leq k \leq M_{2}$. Then, the LHS of (46) simplifies to

$$
\sum_{i=1}^{M_{1}} z_{i}^{2} \sum_{\gamma \delta}^{N}\left(\bar{\eta}_{\gamma}\right)^{m}\left(\bar{\eta}_{\delta}\right)^{n} \prod_{j \neq i}^{M_{1}}\left(\eta_{\gamma}-z_{j}\right)\left(\eta_{\delta}-z_{j}\right) .
$$

Using (E5) we can rewrite (46) as

$$
\begin{aligned}
\sum_{i=1}^{M_{1}} z_{i}^{2} & \left.\left.\mathcal{S}^{M_{1}-1}\left(z_{1} \cdots z_{M_{1}-m-1}\right)\right|_{\text {no } z_{i}} \mathcal{S}^{M_{1}-1}\left(z_{1} \cdots z_{M_{1}-n-1}\right)\right|_{\text {no } z_{i}} \\
= & \left(M_{1}-m\right) \mathcal{S}^{M_{1}}\left(z_{1} \cdots z_{M_{1}-m}\right) \mathcal{S}^{M_{1}}\left(z_{1} \cdots z_{M_{1}-n}\right) \\
& -\sum_{\ell=1}^{\ell_{\mathrm{m}}}(m-n+2 \ell) \mathcal{S}^{M_{1}}\left(z_{1} \cdots z_{M_{1}-m-\ell}\right) \mathcal{S}^{M_{1}}\left(z_{1} \cdots z_{M_{1}-n+\ell}\right) .
\end{aligned}
$$


As all terms in $(\underline{\mathrm{F} 3})$ are completely symmetric in the $z_{i}$ 's, we simplify the notation by imagining to have written an operator $\mathcal{S}^{M_{1}}$ around each term and then order all terms inside, i.e., we replace

$$
\mathcal{S}^{M}\left(z_{1} \cdots z_{m}\right) \rightarrow\left(\begin{array}{c}
M \\
m
\end{array}\right) z_{1} \cdots z_{m}
$$

The remainder of the proof makes use of the auxiliary theorem

\section{Lemma 5}

$$
\mathcal{S}^{M}\left(z_{1} \cdots z_{m}\right) \mathcal{S}^{M}\left(z_{1} \cdots z_{n}\right) \rightarrow \sum_{k=k_{\min }}^{k_{\max }}\left(\begin{array}{c}
M \\
m
\end{array}\right)\left(\begin{array}{c}
m \\
k
\end{array}\right)\left(\begin{array}{c}
M-m \\
n-k
\end{array}\right)[k, m+n-k],
$$

where $k_{\min }=\max (0, m+n-M), k_{\max }=\min (m, n)$, and $[k, m+n-k]=$ $z_{1}^{2} \cdots z_{k}^{2} z_{k+1} \cdots z_{m+n-k}$.

Proof: Replace first the LHS as

$$
\mathcal{S}^{M}\left(z_{1} \cdots z_{m}\right) \mathcal{S}^{M}\left(z_{1} \cdots z_{n}\right) \rightarrow\left(\begin{array}{c}
M \\
m
\end{array}\right) z_{1} \cdots z_{m} \mathcal{S}^{M}\left(z_{1} \cdots z_{n}\right)
$$

In order to obtain the contribution $z_{1}^{2} \cdots z_{k}^{2}, k$ coordinates out of $z_{1}, \ldots, z_{n}$ have to match $k$ coordinates out of $z_{1}, \ldots, z_{m}$, which yields $\left(\begin{array}{c}m \\ k\end{array}\right)$ terms for $k_{\min } \leq k \leq k_{\max }$. The remaining $n-k$ coordinates out of $z_{1}, \ldots, z_{n}$ match $z_{m+1}, \ldots, z_{m+n-k}$, which yields $\left(\begin{array}{c}M-m \\ n-k\end{array}\right)$ terms.

Now, by application of this lemma the first term on the RHS of (F3) simplifies to

$$
\left(M_{1}-m\right) \sum_{k=k_{\mathrm{m}}}^{M_{1}-m}\left(\begin{array}{c}
M_{1} \\
m
\end{array}\right)\left(\begin{array}{c}
M_{1}-m \\
k
\end{array}\right)\left(\begin{array}{c}
m \\
M_{1}-n-k
\end{array}\right)\left[k, 2 M_{1}-m-n-k\right],
$$

where $k_{\mathrm{m}}=\max \left(0, M_{1}-m-n\right)$ and we have used $n \leq m$. The second term on the RHS of (F3) reads

$$
-\sum_{\ell=1}^{\ell_{\mathrm{m}}} \sum_{k=k_{\mathrm{m}}}^{M_{1}-m-\ell}(m-n+2 \ell)\left(\begin{array}{c}
M_{1} \\
m+\ell
\end{array}\right)\left(\begin{array}{c}
M_{1}-m-\ell \\
k
\end{array}\right)\left(\begin{array}{c}
m+\ell \\
M_{1}-n+\ell-k
\end{array}\right)\left[k, 2 M_{1}-m-n-k\right] .
$$

In this equation we change the order of the summations according to

$$
\sum_{\ell=1}^{\ell_{\mathrm{m}}} \sum_{k=k_{\mathrm{m}}}^{M_{1}-m-\ell} \rightarrow \sum_{k=k_{\mathrm{m}}}^{M_{1}-m-1} \sum_{\ell=1}^{M_{1}-m-k}
$$

On the LHS of (F3) at least $z_{1}^{2}$ is present and hence we find

$$
M_{1} \sum_{k=k_{\mathrm{m}}^{\prime}}^{M_{1}-m}\left(\begin{array}{c}
M_{1}-1 \\
m
\end{array}\right)\left(\begin{array}{c}
M_{1}-m-1 \\
k-1
\end{array}\right)\left(\begin{array}{c}
m \\
M_{1}-n-k
\end{array}\right)\left[k, 2 M_{1}-m-n-k\right]
$$


where $k_{\mathrm{m}}^{\prime}=\max \left(1, M_{1}-m-n\right)$. By considering each $k$ separately, (ㅍ3 $)$ simplifies to

$$
\begin{aligned}
\frac{k}{\left(M_{1}-m-k\right) !\left(M_{1}-n-k\right) !} & =\frac{M_{1}-m}{\left(M_{1}-m-k\right) !\left(M_{1}-n-k\right) !} \\
& -\sum_{\ell=1}^{M_{1}-m-k} \frac{m-n+2 \ell}{\left(M_{1}-m-k-\ell\right) !\left(M_{1}-n-k+\ell\right) !} .
\end{aligned}
$$

For $k=M_{1}-m$, (F5) is trivially satisfied. For $\max \left(0, M_{1}-m-n\right) \leq k \leq M_{1}-m-1$, (F5) reduces to

$$
\frac{m}{m ! n !}=\sum_{\ell=1}^{m} \frac{n-m+2 \ell}{(m-\ell) !(n+\ell) !},
$$

which is easily verified. This completes the proof of Theorem 4.

[1] M. Isobe and Y. Ueda, J. Phys. Soc. Jpn. 65, 1178 (1996).

[2] Y. Fujii, H. Nakao, T. Yosihama, M. Nishi, K. Nakajima, K. Kakurai, M. Isobe, Y. Ueda, and H. Sawa, J. Phys. Soc. Jpn. 66, 326 (1997).

[3] Y. Kitaoka, T. Kobayashi, A. Kōda, H. Wakabayashi, Y. Niino, H. Yamakage, S. Taguchi, K. Amaya, K. Yamaura, M. Takano, A. Hirano, and R. Kanno, J. Phys. Soc. Jpn. 67, 3703 (1998).

[4] Y. Tokura and N. Nagaosa, Science 288, 462 (2000).

[5] Y. Q. Li, M. Ma, D. N. Shi, and F. C. Zhang, Phys. Rev. Lett. 81, 3527 (1998).

[6] B. Frischmuth, F. Mila, and M. Troyer, Phys. Rev. Lett. 82, 835 (1999).

[7] F. Mila, B. Frischmuth, A. Deppeler, and M. Troyer, Phys. Rev. Lett. 82, 3697 (1999).

[8] P. Azaria, A. O. Gogolin, P. Lecheminant, and A. A. Nersesyan, Phys. Rev. Lett. 83, 624 (1999).

[9] M. van den Bossche, P. Azaria, P. Lecheminant, and F. Mila, Phys. Rev. Lett. 86, 4124 (2001).

[10] C. Honerkamp and W. Hofstetter, Phys. Rev. Lett. 92, 170403 (2004).

[11] R. Assaraf, P. Azaria, E. Boulat, M. Caffarel, and P. Lecheminant, Phys. Rev. Lett. 93, 016407 (2004).

[12] E. R. I. Abraham, W. I. McAlexander, J. M. Gerton, R. G. Hulet, R. Côté, and A. Dalgarno, Phys. Rev. A 55, R3299 (1997).

[13] C. A. Regal and D. S. Jin, Phys. Rev. Lett. 90, 230404 (2003).

[14] D. Schuricht and M. Greiter, Europhys. Lett. 71, 987 (2005). 
[15] F. D. M. Haldane, Phys. Rev. Lett. 60, 635 (1988).

[16] B. S. Shastry, Phys. Rev. Lett. 60, 639 (1988).

[17] F. D. M. Haldane, Phys. Rev. Lett. 66, 1529 (1991).

[18] F. D. M. Haldane, Z. N. C. Ha, J. C. Talstra, D. Bernard, and V. Pasquier, Phys. Rev. Lett. 69, 2021 (1992).

[19] N. Kawakami, Phys. Rev. B 46, 1005 (1992).

[20] N. Kawakami, Phys. Rev. B 46, R3191 (1992).

[21] Z. N. C. Ha and F. D. M. Haldane, Phys. Rev. B 46, 9359 (1992).

[22] Z. N. C. Ha and F. D. M. Haldane, Phys. Rev. B 47, 12459 (1993).

[23] F. Wilczek, Fractional statistics and anyon superconductivity (World Scientific, 1990).

[24] F. D. M. Haldane, Phys. Rev. Lett. 67, 937 (1991).

[25] H. Georgi, Lie Algebras in Particle Physics (Addison-Wesley, Redwood City, 1982).

[26] V. Chari and A. Pressley, A Guide to Quantum Groups (Cambridge University Press, Cambridge, 1998).

[27] P. Bouwknegt and K. Schoutens, Nucl. Phys. B482, 345 (1996).

[28] K. Schoutens, Phys. Rev. Lett. 79, 2608 (1997).

[29] T. Yamamoto, Y. Saiga, M. Arikawa, and Y. Kuramoto, Phys. Rev. Lett. 84, 1308 (2000).

[30] T. Yamamoto, Y. Saiga, M. Arikawa, and Y. Kuramoto, J. Phys. Soc. Jpn. 69, 900 (2000).

[31] Y. Kuramoto and Y. Kato, J. Phys. Soc. Jpn. 64, 4518 (1995).

[32] Y. Kato and Y. Kuramoto, J. Phys. Soc. Jpn. 65, 1622 (1996).

[33] P. Bouwknegt and K. Schoutens, Nucl. Phys. B547, 501 (1999).

[34] M. Greiter and D. Schuricht, manuscript in preparation.

[35] B. A. Bernevig, D. Giuliano, and R. B. Laughlin, Phys. Rev. B 64, 24425 (2001).

[36] B. Sutherland, Phys. Rev. A 4, 2019 (1971).

[37] B. Sutherland, Phys. Rev. A 5, 1372 (1972).

[38] M. Greiter and D. Schuricht, Phys. Rev. B 71, 224424 (2005).

[39] F. H. L. Eßler, Phys. Rev. B 51, 13357 (1995).

[40] M. Greiter, D. Schuricht, and R. Thomale, manuscript in preparation.

[41] N. Andrei, in Low-dimensional quantum field theories for condensed matter physicists, edited by S. Lundquist, G. Morandi, and Y. Lu (World Scientific, Singapore, 1992).

[42] S. Lang, Complex Analysis (Springer, New York, 1985). 